\title{
The Effect of Surface Drag Strength on Mesocyclone Intensification and Tornadogenesis in Idealized Supercell Simulations
}

\author{
BRETT RoBERTs ${ }^{\mathrm{a}}$ AND MING XUE \\ Center for Analysis and Prediction of Storms, and School of Meteorology, University of Oklahoma, Norman, Oklahoma \\ DANIEL T. DAWSON II \\ Purdue University, West Lafayette, Indiana
}

(Manuscript received 24 April 2019, in final form 22 January 2020)

\begin{abstract}
A suite of six idealized supercell simulations is performed in which the surface drag coefficient $C_{d}$ is varied over a range of values from 0 to 0.05 to represent a variety of water and land surfaces. The experiments employ a new technique for enforcing a three-force balance among the pressure gradient, Coriolis, and frictional forces so that the environmental wind profile can remain unchanged throughout the simulation. The initial low-level mesocyclone lowers toward the ground, intensifies, and produces a tornado in all experiments with $C_{d} \geq 0.002$, with the intensification occurring earlier for larger $C_{d}$. In the experiment with $C_{d}=0$, the low-level mesocyclone remains comparatively weak throughout the simulation and does not produce a tornado. Vertical cross sections through the simulated tornadoes reveal an axial downdraft that reaches the ground only in experiments with smaller $C_{d}$, as well as stronger corner flow in experiments with larger $C_{d}$. Material circuits are initialized enclosing the low-level mesocyclone in each experiment and traced backward in time. Circulation budgets for these circuits implicate surface drag acting in the inflow sector of the supercell as having generated important positive circulation, and its relative contribution increases with $C_{d}$. However, the circulation generation is similar in magnitude for the experiments with $C_{d}=0.02$ and 0.05 , and the tornado in the latter experiment is weaker. This suggests the possible existence of an optimal range of $C_{d}$ values for promoting intense tornadoes within our experimental configuration.
\end{abstract}

\section{Introduction}

The role of surface drag in supercell dynamics, and particularly in tornadogenesis, continues to receive heightened research interest during recent years. To a large degree, the present study represents an extension of Roberts et al. (2016, hereafter R16) and Roberts and Xue (2017, hereafter RX17) that examine the effects of surface drag using a fixed drag coefficient $C_{d}$ value of 0.01 . As such, we will first summarize those two studies for context, then briefly review relevant studies over the past few years.

\section{a. Summary of R16 and RX17}

R16 performed a pair of idealized supercell simulation experiments at $50 \mathrm{~m}$ grid spacing using the Advanced

\footnotetext{
${ }^{a}$ Current affiliation: Cooperative Institute for Mesoscale Meteorological Studies, and NOAA/NWS/Storm Prediction Center, and NOAA/OAR/National Severe Storms Laboratory, Norman, Oklahoma.
}

Corresponding author: Ming Xue, mxue@ou.edu
Regional Prediction System (ARPS; Xue et al. 2000, 2001, 2003) initialized with an environmental sounding based on the 3 May 1999 tornado outbreak in central Oklahoma. The first experiment, full-wind friction (FWFRIC), employed the standard ARPS model formulation for surface drag where drag is applied to the full near-surface horizontal wind components. The second experiment, environment-only friction (EnvFRIC), used a modified formulation where drag was effectively applied only to the base-state wind profile; that is, drag acted only to maintain the environmental wind profile that was in three-force balance among the horizontal pressure gradient force (PGF), Coriolis force, and surface drag, and did not influence perturbation winds associated with the simulated storm. Both experiments used a drag coefficient of $C_{d}=0.01$. A strong tornado occurred in FWFRIC about 25 min into the supercell storm simulation, while no tornado occurred in EnvFRIC during the first $40 \mathrm{~min}$. Vorticity budgets along tornado-entering trajectories in FWFRIC revealed strong enhancement of horizontal vorticity by surface drag in the near-ground inflow east 
of the mesocyclone, which subsequently contributed to cyclonic vorticity in the tornado after tilting and stretching when the trajectories turned upward. In addition, nearground horizontal convergence along a boundary beneath the low-level mesocyclone was shown to be substantially stronger in FWFRIC than in EnvFRIC during and preceding tornadogenesis.

RX17 extended the analysis of experiments FWFRIC and EnvFRIC to the low-level mesocyclone during the pretornadogenesis phase of the simulated storm evolution. Lagrangian circulation budget analyses were performed to elucidate the source of circulation along evolving material circuits. The material circuits were initialized around the low-level mesocyclone and traced backward in time. The circulation budgets for the circuits in FWFRIC revealed that, for the low-level mesocyclone below $1 \mathrm{~km}$ AGL during the $5 \mathrm{~min}$ period immediately preceding tornadogenesis, surface drag had generated a substantial fraction of the mesocyclone circulation. The low-level mesocyclone circulation in EnvFRIC during the same period, while comparable in magnitude to that in FWFRIC, was predominantly barotropic in origin (i.e., it originated from the preexisting environmental wind shear). In both simulations, only a weak cold pool with a small footprint had developed during the period preceding tornadogenesis, and baroclinity was shown to make a minimal contribution to the mesocyclone circulation.

Taken in sum, the results of R16 and RX17 illustrated a scenario of supercell evolution wherein surface drag generates enhanced horizontal vorticity on the storm scale, and this vorticity is then tilted and stretched to contribute meaningfully to cyclonic vorticity in the mesocyclone and tornado. One limitation of those results is that only a single value of $C_{d}$ was employed, so the sensitivity of the mesocyclone intensification and tornadogenesis to the drag strength is unknown. For example, will the tornado be increasingly stronger if the drag coefficient is increased to the upper limits associated with real land surfaces, or will sufficiently strong drag weaken or even eliminate the tornado? To answer these questions, the present study performs a suite of idealized supercell simulations in which $C_{d}$ is set to zero or to values between 0.002 and 0.05 ; the nonzero values cover a representative range for drag over water and land surfaces of different roughnesses. To facilitate the use of different $C_{d}$ values in idealized storm simulations within the same environment, a new technique is employed to keep the environmental wind profile in the far field more or less unchanged throughout the simulation regardless of the value of $C_{d}$, while still allowing drag to act on the full wind components. Effects of the drag strength on the simulated storm intensity and structures, especially those pertaining to the mesocyclone and ensuing tornado, are documented. The relative contributions of surface drag to the circulation about mesocyclone-enclosing material circuits for different drag strengths are also analyzed and compared.

\section{b. Recent progress on supercell and tornadogenesis dynamics}

In the period since the preparation of RX17, a theme in much of the new literature on the tornadogenesis problem has been a return to somewhat more fundamental questions about relevant supercell dynamics, rather than a special emphasis on which physical mechanism(s) generate tornadic vorticity. For example, Coffer and Parker (2016, hereafter CP16) examined idealized singlesounding $125 \mathrm{~m}$ numerical simulations initialized with composites of observed soundings collected during the VORTEX2 field project. Specifically, a simulation initialized using a composite of tornadic cases was compared against one initialized using a composite of nontornadic cases. The supercell in the tornadic composite experiment produced a tornado-like vortex (TLV) with EF3-strength wind speeds, while the supercell in the nontornadic composite experiment failed to produce an organized TLV. This outcome was linked to the stronger low-level mesocyclone and associated updraft in the tornadic composite experiment. The relatively more dominant streamwise (crosswise) vorticity near the ground in the tornadic (nontornadic) composite sounding is argued, after tilting and ingest into the low-level mesocyclone, to have more effectively induced a positive feedback of dynamic perturbation pressure falls aloft that gave rise to this robust updraft. Implicit in this conclusion is that the environmental wind shear (and associated barotropic horizontal vorticity) is a crucial and direct control on mesocyclone processes near and below $1 \mathrm{~km}$ AGL, in addition to its more ubiquitously understood impact on midlevel rotation. This is noteworthy because the literature on supercell dynamics has long emphasized the need for downdrafts to generate "near ground" cyclonic vorticity in a supercell. The basis for this argument is typically that tilting of horizontal vorticity in near-ground parcels ascending into an updraft cannot commence quickly enough to generate meaningful vertical vorticity until some appreciable height AGL (e.g., Rotunno and Klemp 1985; Davies-Jones and Brooks 1993; Markowski et al. 2008; Davies-Jones 2015). However, Rotunno et al. (2017, hereafter RMB17) have cast some doubt on this notion, using highly idealized numerical experiments (in which a pseudostorm updraft and downdraft were forced by persistent specified heating and cooling sources, respectively, and the lower boundary was free slip) to demonstrate how near-ground parcels with initially negligible vertical vorticity can in fact acquire cyclonic vorticity 
"near the ground" (e.g., $1 \mathrm{~m}$ AGL) immediately upon ascent. The key ingredient for this near-ground production is the presence of very large streamwise horizontal vorticity prior to ascent; due to the action of stretching, such large streamwise vorticity is more likely to be found in accelerating flows (e.g., an outflow surge, or inflow accelerating toward the center of an intensifying mesocyclone) than decelerating flows (e.g., inflow stagnating upon approach to a strong, vertically erect gust front; Davies-Jones and Markowski 2013). Note that in RMB17's experiments, preexisting cyclonic vorticity prior to ascent is still beneficial for subsequently generating large values near the ground, even if it is not strictly necessary.

R16 identified a mechanism for cyclonic vorticity production during trajectory descent toward the ground analogous to the "vortex line slippage" mechanism in Davies-Jones and Brooks (1993), except that it is the exchange of frictional crosswise vorticity into the horizontal streamwise direction-rather than direct, baroclinic generation of horizontal streamwise vorticity-which initiates the process during descent (cf. Fig. 19 in R16). This mechanism, which was demonstrated for a representative parcel trajectory entering the simulated tornado in R16, is one example of how vorticity generated without baroclinic influence could contribute directly to tornadogenesis. If the arguments of RMB17 apply to most supercells in nature, it is even possible that near-ground parcels without a history of descent could contribute to substantial near-ground cyclonic vorticity in low-level mesocyclones and tornadoes ${ }^{1}$; in principle, this would further open the door to important generation mechanisms other than baroclinity (e.g., frictional generation, or tilting and stretching of environmental vorticity). Considering that frictionally generated horizontal vorticity is largest near the ground, and previous modeling studies (Schenkman et al. 2014; R16) have shown that the so-called riverbend effect can convert much of the initially crosswise frictional vorticity possessed by tornadobound parcels into streamwise vorticity as the flow curves cyclonically and converges toward the vortex (after which tilting into the vertical and amplification from stretching can be expected upon ascent into the vortex), it is clear that further investigation into frictional effects on supercell dynamics and tornadogenesis is warranted.

Coffer and Parker (2018, hereafter CP18) conducted an expanded suite of idealized experiments initialized

\footnotetext{
${ }^{1}$ Although both parcels analyzed in RMB17 descended prior to acquiring cyclonic vorticity, their results demonstrate a key physical mechanism (large stretching of horizontal streamwise vorticity prior to ascent) that could also manifest in parcels accelerating horizontally near the ground without a history of participation in a downdraft.
}

with intermediate soundings interpolated between the CP16 tornadic and nontornadic composite profiles, finding a "tipping point" where TLV genesis occurs if the background environment is composed at least $40 \%$ of the tornadic composite. Again, the role of the low-level environmental horizontal vorticity magnitude and orientation (in particular, the 0-500 m AGL storm-relative helicity) in promoting a robust low-level mesocyclone is identified as the key causal factor for this tipping point. CP18 state among their key conclusions that "operationally, it matters not how vertical vorticity is generated at the surface," so long as a strong low-level updraft exists to stretch the vorticity sufficiently upon ascent. This is a finding echoed by Yokota et al. (2018, hereafter Y18), who conducted a 33-member ensemble of $50 \mathrm{~m}$ real-data simulations of the 6 May 2012 Tsukuba, Japan, supercell tornado case. Circulation analyses for circuits initialized encircling tornadoes in various members, then integrated backward in time, revealed that friction tended to have a larger overall contribution to circulation than baroclinity; nonetheless, baroclinity was a dominant source of tornadic circulation in some members. Furthermore, the relative roles of different circulation generation mechanisms within an ensemble member were not strongly correlated with the existence or intensity of a tornado in that member. Instead, Y18 found "tornadogenesis was especially well correlated with the strength of low-level mesocyclones at about $1 \mathrm{~km}$ AGL and water vapor near the surface" in the minutes prior to genesis. The critical role of the low-level mesocyclone and updraft strength was also seen in RX17 and several other high-resolution modeling studies (e.g., Noda and Niino 2010; Mashiko 2016), with RX17 placing particular emphasis on the effect of horizontal vorticity generated by surface drag on the mesocyclone intensity. Trapp et al. (2017) has argued using a simple mathematical model that the updraft width, in addition to strength, may also control tornado intensity. A commonality of these studies is the critical role played by the low-level mesocyclone, which provides the needed intense vertical stretching. Meanwhile, these studies also point to the important role of the vertical shear/horizontal vorticity contained in the flow feeding the mesocyclone, which may come from the background environment or be generated/enhanced by the storm (e.g., through baroclinity or surface friction). These findings motivate a particular focus on the dynamics and evolution of the low-level mesocyclone in the present study across our suite of experiments with different $C_{d}$ values.

As fundamental conceptual models of supercell behavior are evolving to accommodate new insights and experimental results, identification of specific vorticity generation mechanisms acting on parcels bound for the 
low-level mesocyclone and tornado remains an important facet of understanding their dynamics. Furthermore, in the case of surface drag, any potential role it might play in important vorticity generation has potential operational relevance, since the surface roughness beneath and surrounding a supercell can in many cases be reasonably assessed in real time. Thus, our continued work in this area has both academic and operational relevance.

The rest of this paper is organized as follows. Section 2 briefly describes the new technique for maintaining the environmental wind profile, and how it differs from the experimental setup in R16. Section 3 presents the results of our new experiments. Section 4 summarizes the results, discusses their implications, and suggests possible areas of future research.

\section{Methodology}

\section{a. Environmental wind balance technique}

When compared to experiments FWFRIC and EnvFRIC analyzed in R16 and RX17 (described in section 1a), the simulations analyzed in the present study differ chiefly in our approach to modeling the force balance in the background environment, and also in the actual value of the surface drag coefficient.

In section $2 \mathrm{~b}$ of $\mathrm{R} 16$, we detailed a procedure for establishing a base-state sounding that is in a three-force balance among the horizontal PGF, Coriolis force, and frictional force (the so-called geotriptic balance; Johnson 1966). In the current study, we will refer to the forcebalancing technique from R16 as the frictional balancing procedure (FBP). As a brief review, the FBP involved integrating a $1 \mathrm{D}$ version of the ARPS model (the same model used for the 3D storm simulations, with the same vertical grid spacing and physics parameterizations) for a $48 \mathrm{~h}$ adjustment period. The $1 \mathrm{D}$ simulation was initialized with a sounding extracted from a real-data simulation of the 3 May 1999 tornado outbreak in central Oklahoma conducted by Dawson et al. (2010, hereafter DA10); this initial sounding (called MAY3) was assumed to be in geostrophic balance, even though drag acting within the modeled planetary boundary layer (PBL) qualitatively violated this assumption. The 1D simulation effectively included the large-scale PGF, Coriolis force, and surface drag (using $C_{d}=0.01$, which was selected as an intermediate value representative of land). After the $48 \mathrm{~h}$ adjustment, a three-force balance was achieved in the $1 \mathrm{D}$ column, and the resulting thermodynamic and kinematic profiles were taken as a sounding we called MAY3B (Fig. 1). In R16 and RX17, MAY3B was used to define the storm environment in 3D storm simulation experiments (FWFRIC and EnvFRIC)
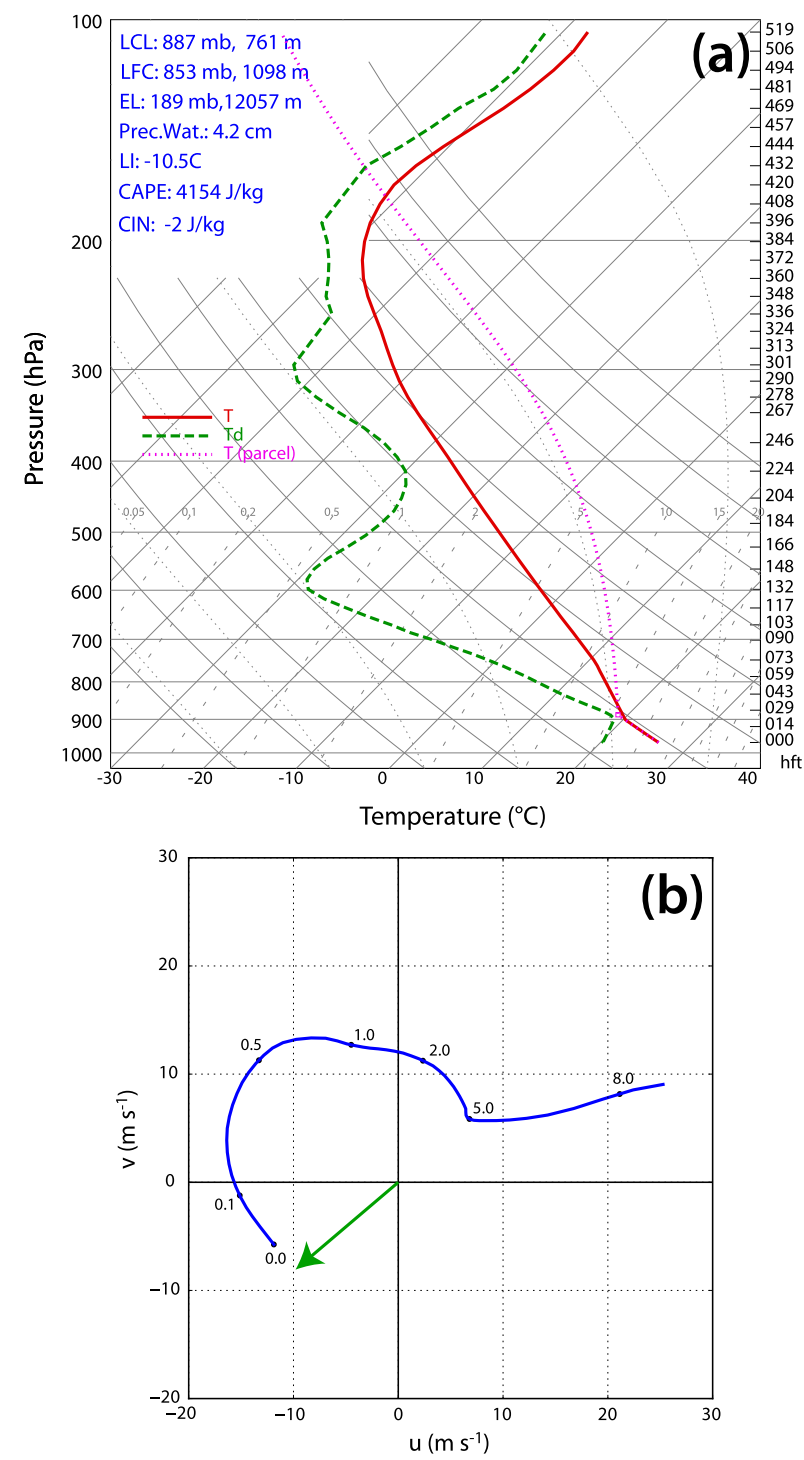

FIG. 1. (a) Skew $T-\log p$ plot and (b) wind hodograph, representing sounding MAY3B, which is used to initialize all experiments in the present study. In (b), dots and their annotations represent heights ( $\mathrm{km} \mathrm{AGL),} \mathrm{and} \mathrm{the} \mathrm{green} \mathrm{arrow} \mathrm{emanating} \mathrm{from}$ the origin represents the "ground-motion vector" (i.e., the vector that is added to the base-state wind profile to obtain a quasistationary storm in the simulation). Adapted from R16.

that used $C_{d}=0.01$. By employing the FBP, R16 and RX17 compared the application of parameterized surface drag to the full wind (FWFRIC) versus its application to only the base-state wind (EnvFRIC). The action of surface drag within the 1D adjustment simulation resulted in a substantial change in the low-level wind profile of MAY3B, when compared to the original MAY3 sounding. MAY3B consequently contains an excess of near-ground shear (e.g., $0-1 \mathrm{~km}$ storm-relative helicity of $435 \mathrm{~m}^{2} \mathrm{~s}^{-2}$ in MAY3B vs $310 \mathrm{~m}^{2} \mathrm{~s}^{-2}$ in MAY3) 
that is attributable directly to the assumption of MAY3's geostrophy in the FBP. Furthermore, this change in lowlevel shear was necessarily dependent on the magnitude of $C_{d}$, meaning that different choices of $C_{d}$ would have yielded different shear profiles. Therefore, with the FBP method, it was not possible to run experiments varying $C_{d}$ while keeping the same environmental wind profile balanced in all of the simulations. It is the goal of this study to overcome this limitation and examine the impact of different $C_{d}$ values on storms developing within the same environment.

In the present study, the FBP is supplanted by a new approach called the geotriptic wind balance (GWB) technique introduced in a companion paper (Dawson et al. 2019, hereafter DRX19). Briefly, its purpose is to make practical the use of any sounding to define the storm environment for idealized simulations with surface drag using a constant drag coefficient $C_{d}$. It accomplishes this by explicitly adding a compensating horizontally uniform force to the horizontal momentum equations that balances the Coriolis and friction forces on the large scale (i.e., for the unperturbed storm environment). This compensating force [hereafter the "pseudo-PGF" (PPGF)] is found by evaluating the time tendency of the horizontal momentum equations under the action of Coriolis and frictional forces, typically using a suitable horizontal average of an unperturbed region of the domain near the beginning of the simulation. The required force is then the negative of this time tendency. The PPGF so computed is added immediately back to the RHS of the horizontal momentum equations for the initial time step and all subsequent times. In this manner, the GWB technique will ensure the background environment (i.e., the far field away from storm-induced perturbations) remains in three-force balance, and therefore quasi-steady state, for any background sounding.

Along with this property, another advantage of the GWB technique over the FBP technique makes it ideal for the purposes of the study: while the FBP is only physically justifiable to the extent that the initial input sounding is in geostrophic balance, this is not the case when using the GWB technique, since it explicitly assumes the initial profile is in three-force balance (i.e., Coriolis, PGF, and friction) and is thus more flexible. We refer the reader to DRX19 for further details. In the present study, we apply a range of $C_{d}$ values to experiments that all share the same initial sounding (MAY3B). Thus, in these experiments, we do not claim to model the three-force balance from the real storm environment that produced MAY3B. Instead, as in R16 and RX17, we are artificially forcing the background environment to remain the same over different surfaces in order to discern the impact of drag specifically on storm perturbations.

\section{b. Experimental design and model configuration}

In this study, six experiments with different drag coefficient values are performed using the GWB technique. For continuity with FWFRIC and EnvFRIC (from R16 and RX17; more details in section 1a) and to facilitate clean comparisons with those earlier experiments, the initial sounding for all experiments herein is MAY3B (Fig. 1). We therefore again emphasize the caveat that MAY3B contains some degree of artificially enhanced near-ground shear when compared with DA10's original simulation, as described in section 2 a.

The experiments and their drag coefficients are summarized in Table 1, along with representative land surfaces for each coefficient. ${ }^{2}$ These experiments are intended to sample the parameter space spanned by land surfaces over which supercells may exist in the real world, ranging from short grassland to tall forests and urban cores (with the exception of $\mathrm{CD} 0$, which represents an idealized frictionless surface). Experiment CD0 is the GWB-based equivalent to EnvFRIC; that is, drag does not act on storm perturbations in $\mathrm{CD} 0$, even though its background wind profile has resulted from drag. In the remaining experiments, drag does act on the storm perturbations, but the magnitude varies according to $C_{d}$. Experiment CD1-2, with $C_{d}=0.01\left(1 \times 10^{-2}\right)$, is the GWB-based equivalent to FWFRIC. Although CD0 (CD1-2) is not identical in evolution to EnvFRIC (FWFRIC), they are qualitatively very similar throughout the analysis period.

Our numerical simulations are conducted using the ARPS (Xue et al. 2000, 2001) with the same configuration described in R16, aside from the implementation of the GWB technique and our specified values of $C_{d}$. The grid spacing is $50 \mathrm{~m}$ in the horizontal. There are $83 \mathrm{ver}$ tical levels, and vertical grid spacing ranges from $20 \mathrm{~m}$ near the ground to $400 \mathrm{~m}$ in the upper troposphere. The physical domain is $64 \mathrm{~km} \times 96 \mathrm{~km}$ in horizontal extent and $16 \mathrm{~km}$ deep in the vertical. For this study, simulations were integrated in time to $3000 \mathrm{~s}$. The initial condition is horizontally homogeneous (defined by the aforementioned sounding MAY3B) except for an ellipsoidal thermal bubble with a maximum potential temperature perturbation of $6 \mathrm{~K}$ (used to initiate deep moist convection). Parameterization of microphysics follows the five-species formulation of Lin et al. (1983), but with the rain intercept parameter $n_{0 r}$ reduced to $2 \times 10^{6}$. The 1.5-order TKE formulation of Moeng and Wyngaard (1988) is employed to parameterize subgrid-scale turbulence.

\footnotetext{
${ }^{2}$ Note that only roughness length $z_{0}$ can be linked directly to land surface types, while $C_{d}$ in a numerical model is a function of both $z_{0}$ and the height of the lowest scalar grid level $z_{1}$ (10 m AGL, in our configuration). See (4) of Wieringa (1993) for details.
} 
TABLE 1. Drag coefficients $C_{d}$ for GWB experiments. For each $C_{d}$, the equivalent roughness length $z_{0}$ and representative realworld surface type(s) are presented in accordance with the descriptions of Wieringa (1993).

\begin{tabular}{llcl}
\hline \hline Expt & \multicolumn{1}{c}{$C_{d}$} & Equivalent $z_{0}(\mathrm{~m})$ & Representative surface type \\
\hline CD0 & 0 & 0 & Idealized frictionless \\
CD2-3 & 0.002 & 0.002 & Short grass \\
CD5-3 & 0.005 & 0.04 & Long grass \\
CD1-2 & 0.01 & 0.2 & Cropland \\
CD2-2 & 0.02 & 0.6 & Bushland, suburb \\
CD5-2 & 0.05 & 1.7 & Mature forest, city core \\
\hline
\end{tabular}

\section{Simulation results}

\section{a. Overview and qualitative analysis}

As with the original FWFRIC and EnvFRIC experiments from R16 (described at length in section 1a), all six experiments evolve qualitatively similarly to each other for the first $600 \mathrm{~s}$. Subsequently, as with those two experiments, subtle differences in the near-ground wind field begin to grow during the $600-1200$ s period, yielding more qualitatively meaningful differences by $1500 \mathrm{~s}$. Figure $2 \mathrm{a}$ presents a time series of domainwide ${ }^{3}$ minimum perturbation pressure for the GWB experiments. All experiments with drag enabled $\left(C_{d}>0\right)$ exhibit large pressure deficits of $40-80 \mathrm{hPa}$ during the $1500-2200 \mathrm{~s}$ time period. A tendency exists for an experiment's largest deficit to occur earlier as $C_{d}$ increases (e.g., CD2-2 reaches its minimum around $1500 \mathrm{~s}$, whereas CD2-3 reaches its minimum around $2000 \mathrm{~s}$ ). CD0 stands in stark contrast to the drag-enabled experiments, with pressure deficits remaining smaller than $25 \mathrm{hPa}$ throughout the period. Among the drag-enabled experiments, pressure deficits in CD5-2 are substantially smaller than in the other experiments. A time series of maximum stormrelative horizontal winds (Fig. 2b) also reveals that wind maxima tend to be larger, and occur earlier in time, in the experiments with larger $C_{d}$ (except for the largest value). The differences in maximum wind magnitude between the strong-drag and weak-drag experiments are somewhat less pronounced than the corresponding differences in pressure deficits, however, as wind speeds associated with strong outflows and other nontornadic features can also become quite large (e.g., even CD0 reaches a maximum of $80 \mathrm{~m} \mathrm{~s}^{-1}$ around $2800 \mathrm{~s}$, and this strong flow is not associated with a near-surface vortex; Fig. 2c). A corresponding time series of maximum vertical vorticity below $2 \mathrm{~km}$ AGL (Fig. 2c) tracks the

\footnotetext{
${ }^{3}$ Note that in Fig. 2, although plotted values are domainwide extrema, larger magnitudes are almost always associated with the low-level mesocyclone region and/or tornado.
}

interexperiment timing and magnitude differences of the perturbation pressure minima quite closely, including the relatively weak maxima in CD5-2 when compared to the other drag-enabled experiments. The storm features responsible for these discrepancies will now be shown and discussed.

Figure 3 presents domainwide time-height cross sections from 0 to $3000 \mathrm{~s}$ of maximum updraft and vertical vorticity for the six experiments. The initial lowering of the mesocyclone from around $1500 \mathrm{~m}$ AGL toward the ground can be seen in the plots of updraft magnitude (Figs. 3a-f) to begin earlier during the simulation as $C_{d}$ increases. Similar to FWFRIC in R16, large cyclonic vorticity develops quickly upward from the ground in all experiments except CD0 during the 1300-1800s period (Figs. 3g-1). This process occurs progressively earlier with increasing $C_{d}$ from 0.002 in CD2-3 to 0.02 in CD2-2, but there is little difference in timing between CD2-2 and CD5-2. Based on these cross sections, it appears that surface drag (with a $C_{d}$ value as small as 0.002 ) is required in order for an intense low-level mesocyclone to develop during this early stage of the simulation, and that larger values generally hasten this process. However, at the high end of the sampled $C_{d}$ parameter space, there exist signs of an upper limit on favorability for intense lowlevel mesocyclogenesis somewhere in the range $0.02 \leq$ $C_{d} \leq 0.05$. Although the lowering of the mesocyclone occurs slightly earlier in CD5-2 than in CD2-2, the maximum mesocyclone updraft and vorticity are weaker overall in CD5-2, and intense rotation $\left(\zeta \geq 0.75 \mathrm{~s}^{-1}\right)$ does not extend above $300 \mathrm{~m}$ AGL (Figs. 3d,e,i,j). This may be due to the increasingly large damping effect on the near-surface flow as the surface drag increases in strength.

Horizontal cross sections of horizontal convergence, perturbation pressure, and ground-relative wind vectors at $1320 \mathrm{~s}$ are presented in Fig. 4. In CD0, a broad zone of convergence is seen along the surface boundary, which is primarily north-south oriented and separates westerly and easterly flow (Fig. 4a). As $C_{d}$ increases in the remaining experiments, a few trends are noted. First, the surface boundary becomes progressively more curved along its northern extent around ( $x=36 \mathrm{~km}, y=65 \mathrm{~km})$. Second, the convergence zone becomes more compact, with a larger maximum convergence magnitude at its center (except in CD5-2, where maximum convergence is weaker than in all other drag-enabled experiments). Third, the "inflow low" (denoted by the innermost perturbation pressure contour) east of the boundary becomes centered more toward the northwest. In CD2-2 and CD5-2, a strong pressure minimum associated with a developing tornado can already be seen near $(x=36 \mathrm{~km}$, $y=64 \mathrm{~km}$ ) (Figs. 4e,f). All of these trends largely mirror 

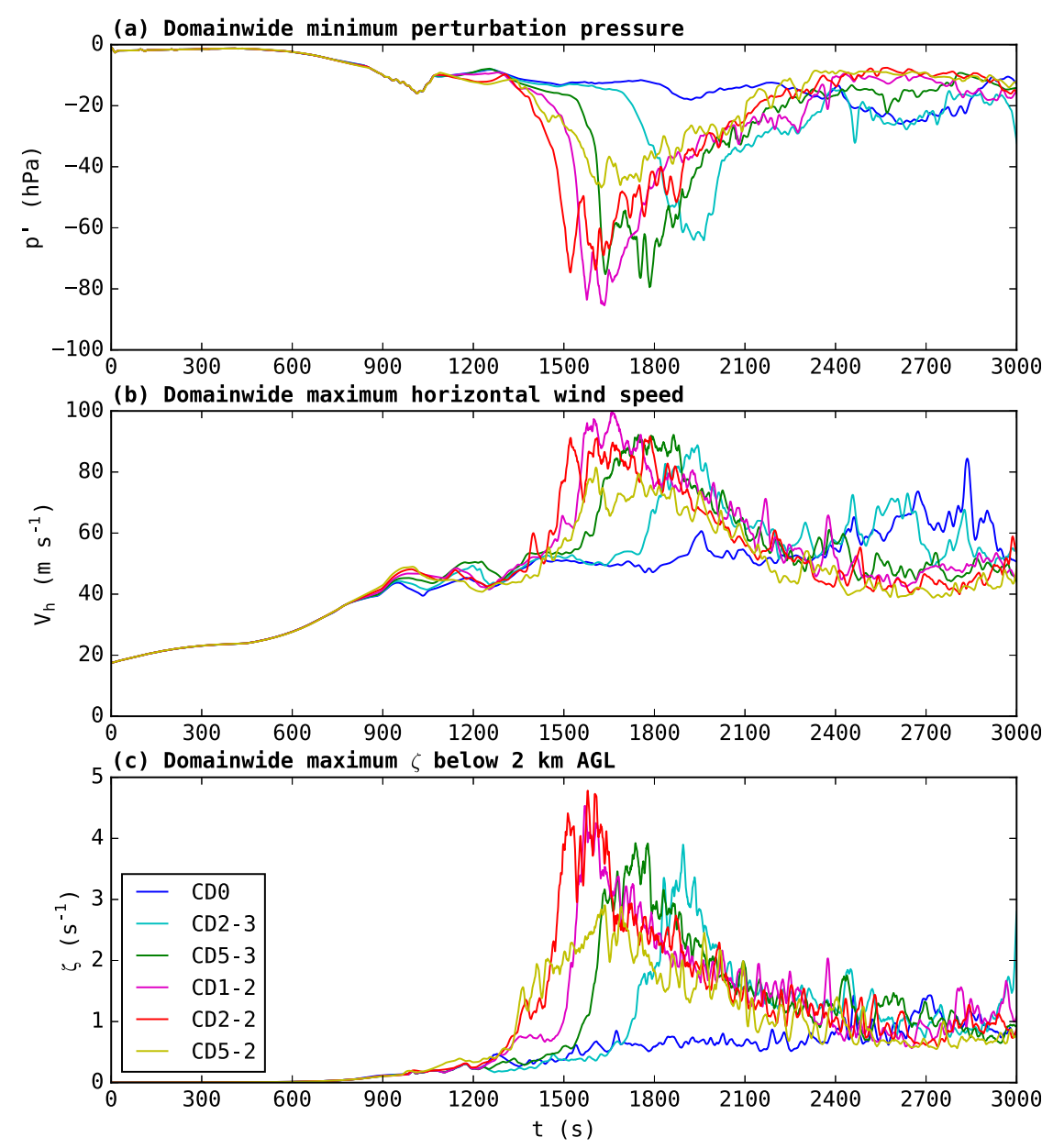

FIG. 2. Time series of domainwide (a) minimum perturbation pressure, (b) maximum horizontal storm-relative wind speed, and (c) maximum vertical vorticity below $2 \mathrm{~km}$ AGL for the six GWB-based experiments between 0 and $3000 \mathrm{~s}$.

the discrepancies between EnvFRIC and FWFRIC analyzed in R16 (see their Fig. 4). The relatively orderly changes with increasing $C_{d}$ between CD0 and CD2-2 bolster confidence that the early-simulation convergence boundary behavior in FWFRIC and EnvFRIC is predictable and representative of monotonic trends within the $C_{d}$ parameter space. By contrast, the markedly weaker convergence maximum in CD5-2 relative to CD2-2 is another indication that surface drag in CD5-2 is so strong as to interfere ${ }^{4}$ with processes that encourage more intense low-level mesocyclogenesis and tornadogenesis during this period in CD2-2 (Figs. 4e,f). The ground-relative flow on both sides of the boundary, and particularly within the inflow east of the boundary, is so weak in CD5-2 (Fig. 4f) that low-level convergence is

\footnotetext{
${ }^{4}$ A tornado occurs shortly after this time in both CD2-2 and CD5-2, but its intensity is much greater in CD2-2 (cf. Fig. 2).
}

relatively anemic, and this proves detrimental to lowlevel updraft maintenance (Fig. 3e).

Figure 5 displays horizontal cross sections at $10 \mathrm{~m}$ AGL and $1800 \mathrm{~s}$, revealing the extent and strength of the surface cold pool and tornado (except in CD0, where no tornado is ongoing at $1800 \mathrm{~s}$ ). At $1800 \mathrm{~s}$, the surface convergence boundary remains more north-south oriented in experiments with smaller $C_{d}$, whereas experiments with larger $C_{d}$ tend to exhibit a strongly curved boundary that wraps into the tornado. It is noteworthy that relatively warm air resulting from a dynamically driven downdraft south of the mesocyclone [e.g., centered near ( $x=32 \mathrm{~km}, y=63 \mathrm{~km})$ in Fig. 5a] tends to wrap cyclonically around the mesocyclone and partially encircle the tornado in the experiments with larger $C_{d}$, whereas the surface boundary south of the mesocyclone in $\mathrm{CD} 0$ (and, to a much lesser extent, CD2-3) appears to block this warm air from wrapping in. 

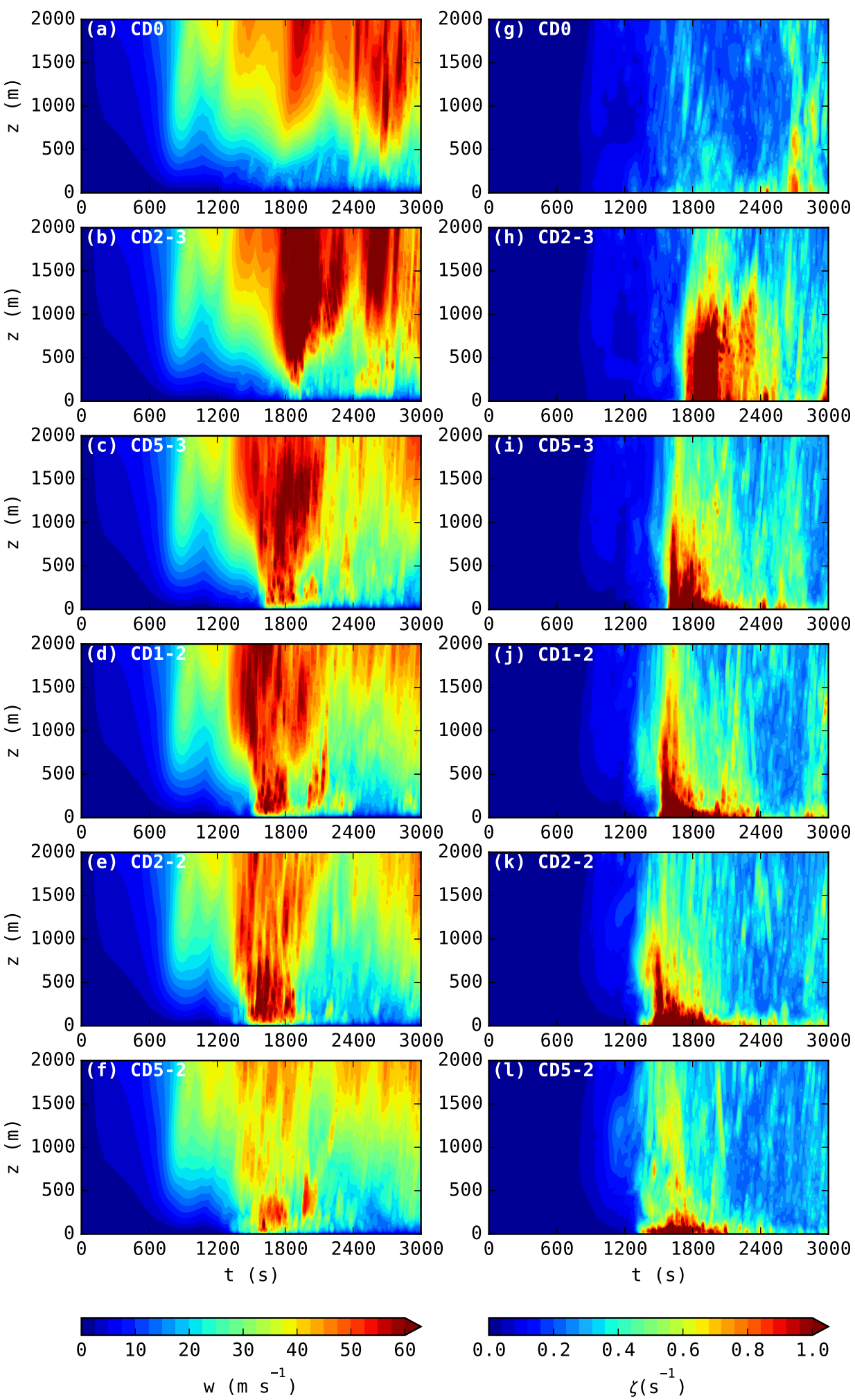

FIG. 3. Domainwide maximum time-height cross sections from 0 to $3000 \mathrm{~s}$ of (left) updraft for (a) CD0, (b) CD2-3, (c) CD5-3, (d) CD1-2, (e) CD2-2, and (f) CD5-2; and (right) vertical vorticity for (g) CD0, (h) CD2-3, (i) CD5-3, (j) CD1-2, (k) CD2-2, and (1) CD5-2. 

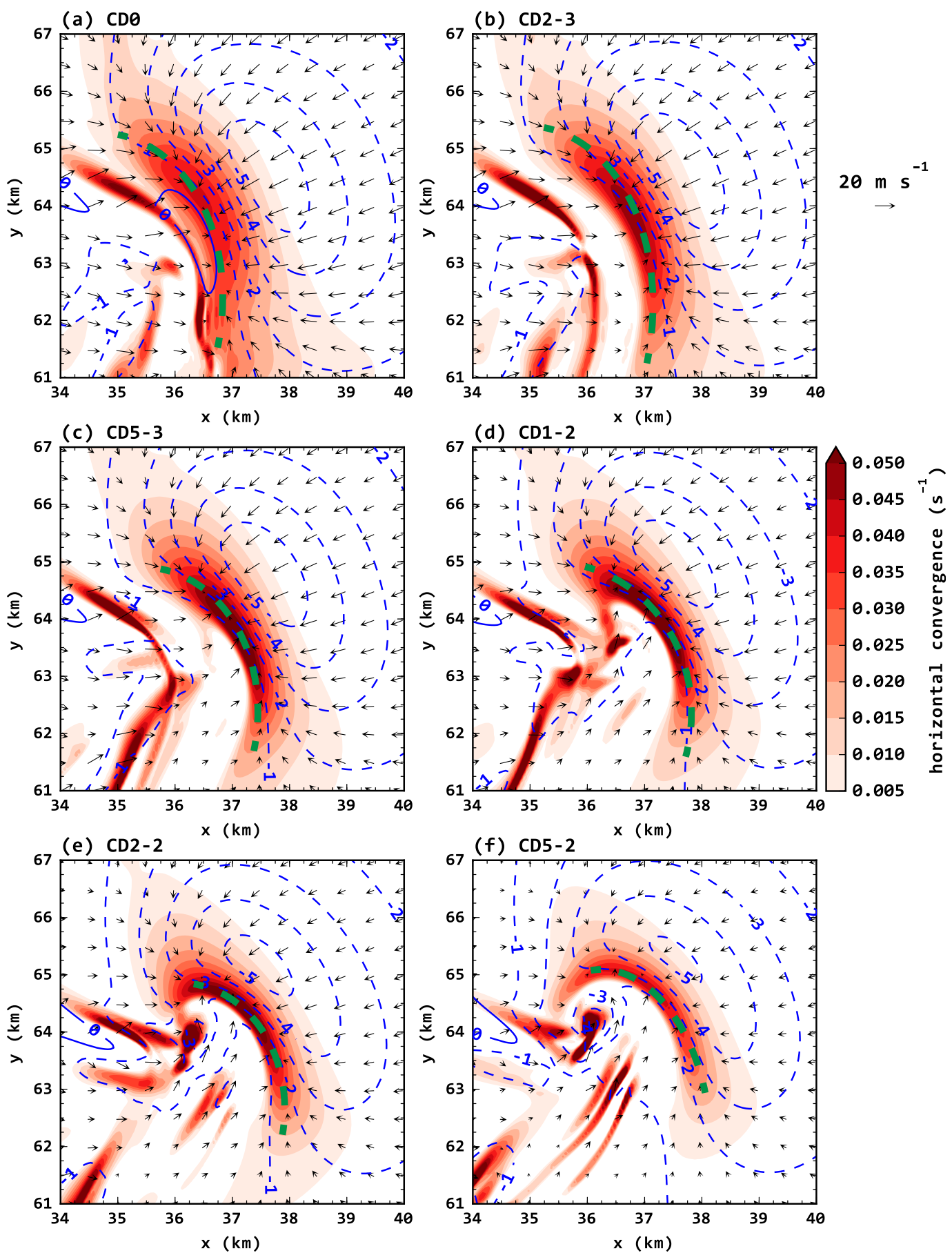

FIG. 4. Horizontal cross section at $10 \mathrm{~m}$ AGL and $1320 \mathrm{~s}$ of horizontal convergence (shaded), perturbation pressure (blue dashed contours every $1 \mathrm{hPa}$ for $p^{\prime} \leq-1 \mathrm{hPa}$ ), and ground-relative wind vectors for (a) CD0, (b) CD2-3, (c) CD5-3, (d) CD1-2, (e) CD2-2, and (f) CD5-2. In each panel, the convergence boundary is annotated with a green dashed curve.

As discussed previously in R16 and RX17, the timing of tornadogenesis in our experiments (about 25-35 min after the introduction of a thermal bubble to induce an updraft) is quite early in the parent storm's life cycle.
The 3D numerical experiments of Markowski (2016) with predominantly crosswise near-ground environmental vorticity exhibited similarly rapid genesis of a tornado-like vortex, although his experiments were dry and used a 

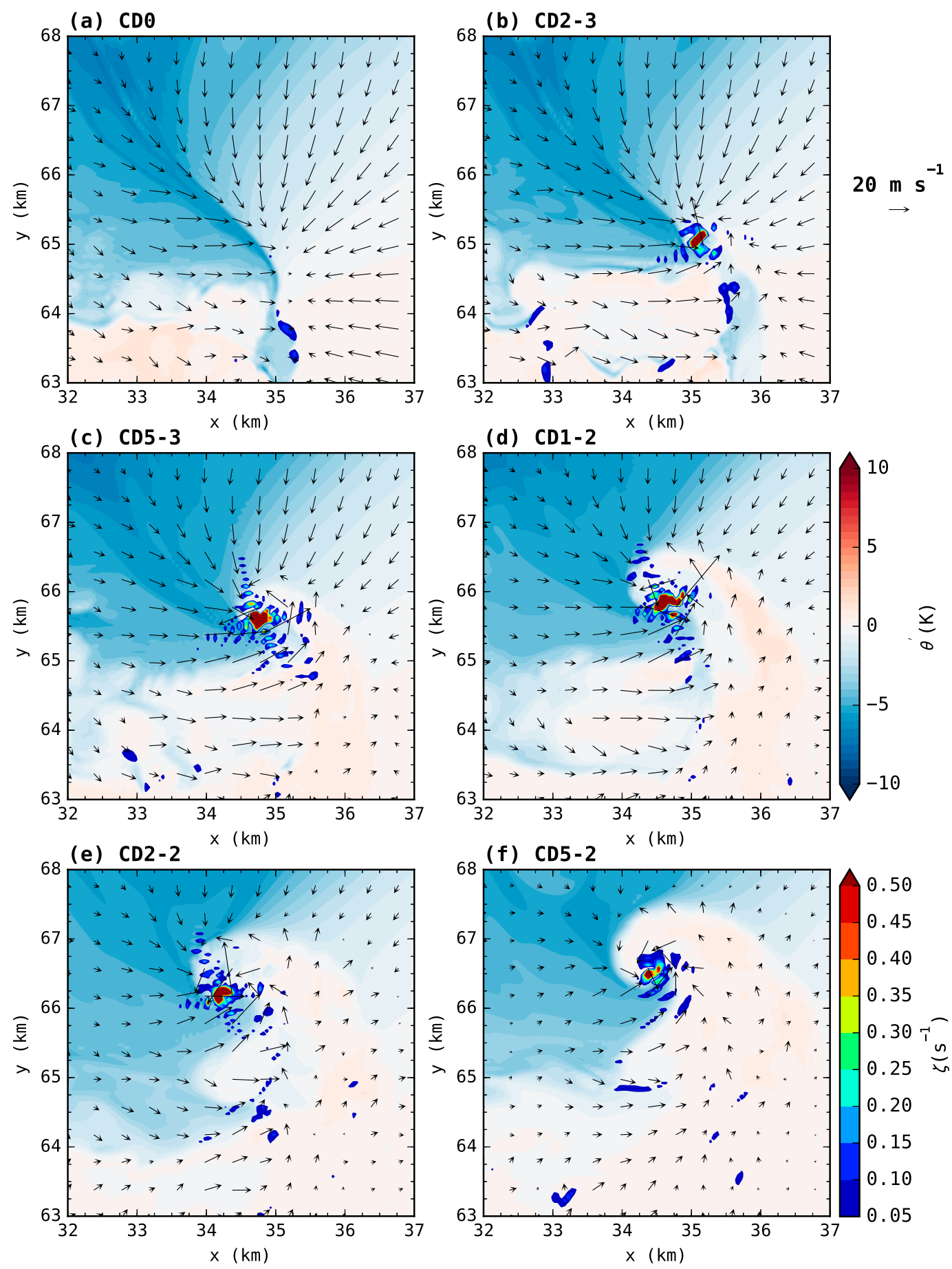

FIG. 5. Horizontal cross section at $10 \mathrm{~m} \mathrm{AGL}$ and $1800 \mathrm{~s}$ of perturbation potential temperature (shaded), cyclonic vorticity (shaded for $\zeta \geq 0.05 \mathrm{~s}^{-1}$ ), and ground-relative wind vectors for (a) CD0, (b) CD2-3, (c) CD5-3, (d) CD1-2, (e) CD2-2, and (f) CD5-2.

much more idealized setup than those in the present study (e.g., while using generally realistic supercell wind profiles, his "pseudostorms" were forced and modulated by an artificial heat source and sink that produced an updraft and downdraft). While a few comparable cases of very rapid tornadogenesis following convective initiation have been documented in real observations (e.g., Palmer et al. 2011; Boustead and Gross 2016), the 
preponderance of evidence suggests most tornadoes forming via supercell mesocyclone processes occur later into the storm life cycle-and with a cooler, more expansive rear-flank downdraft (RFD) adjacent to the lowlevel rotation (e.g., Lemon and Doswell 1979; Markowski 2002), providing greater opportunity for the influence of baroclinic vorticity (e.g., Klemp and Rotunno 1983; Rotunno and Klemp 1985; Markowski et al. 2008)—when compared to our simulations herein. Thus, there is reason for caution in broadly applying conclusions regarding the precursors, dynamics, and evolution of our simulated tornadoes and low-level mesocyclones to their counterparts in real-world supercells. As in R16 and RX17, we stress that our findings through the remainder of this section should be interpreted as evidence of the physical plausibility of dynamically similar vortices within supercells, rather than as necessarily representative of all (or even most) supercell tornadoes in nature. Indeed, extensions of our simulations herein to $4800 \mathrm{~s}$ exhibit a second period of tornado development after a significant cold pool becomes established (not shown); baroclinic vorticity generation is expected to play a larger role alongside frictional generation under such conditions. These results will be analyzed and reported in future work.

\section{b. Tornado structure}

Next, we examine how the tornado-scale structure varies among our experiments, to the extent it is resolved on our grid. Figure 6 presents pseudovertical cross sections of vertical velocity, vertical vorticity, and wind vectors through the first tornado occurring in the drag-enabled experiments (CD2-3, CD5-3, CD1-2, CD2-2, and CD5-3; note that CD0 is excluded in this section because it does not produce a tornado). At each vertical grid level, a horizontal slice of grid points along the $x$ axis is extracted along the $y$ coordinate containing the local minimum in $p^{\prime}$; these linear slices are then stacked vertically to produce the pseudovertical sections in Fig. 6. Effectively, this means that the cross section tilts meridionally with height to keep the tornado center within the cross-section plane. Although the cross sections are taken near the time of peak tornado intensity (as defined by the minimum pressure deficit) in each experiment, it must be cautioned that some discrepancies between panels may be time dependent and/or associated with storm-scale differences not directly tied to the vortex's interaction with the lower boundary; for this reason, we will present more spatiotemporally general statistics below. Nonetheless, the "corner flow" (Rotunno 1977; Lewellen et al. 2000) is more pronounced in CD2-2 and CD5-2 (Figs. 6d,e) than in CD2-3 and CD5-3 (Figs. 6a,b). Consequently, strong $\left(>30 \mathrm{~m} \mathrm{~s}^{-1}\right)$ updraft within the vortex tends to extend downward closer to the ground in experiments with larger $C_{d}$. In CD2-3, which uses the smallest $C_{d}$ among the drag-enabled experiments, the tornado's primary updraft is elevated and fed by flow that turns upward with a relatively large curvature radius in the $x-z$ plane; $w>30 \mathrm{~m} \mathrm{~s}^{-1}$ only occurs above $400 \mathrm{~m}$ AGL (Fig. 6a).

Evidence of marginally resolved multivortex structure near the ground exists to varying degrees in CD5-3 (Fig. 6b), CD1-2 (Fig. 6c), and CD2-2 (Fig. 6d); by contrast, the tornado in CD5-2 features a core axial updraft at the lowest grid levels AGL, supported by horizontal flow there converging sharply from the east and west (Fig. 6e). For context, horizontal cross sections through the vortex at $50 \mathrm{~m}$ AGL of vertical velocity, perturbation pressure, and ground-relative wind vectors are presented in Fig. 7. Downdraft exists at or near the tornado center in CD2-3 (Fig. 7a), CD5-3 (Fig. 7b), and CD1-2 (Fig. 7c); by contrast, updraft dominates the entire inner vortex at this height in CD2-2 (Fig. 7d) and CD5-2 (Fig. 7e).

The trends with respect to corner flow and tornadoscale variations in $w$ seen within our $C_{d}$ parameter space broadly agree with Trapp (2000, hereafter T00), who performed idealized axisymmetric vortex simulations with free-slip and no-slip lower boundary conditions. A key finding in T00 was that an axial (central) downdraft penetrated to the surface almost immediately after vortex genesis in their free-slip simulation, but was dislodged aloft in their no-slip simulation by an intense axial jet erupting upward from the ground. Radial inflow resulting from surface friction disrupting cyclostrophic balance gives rise to this axial jet (Bluestein 2007); with all other variables held constant, larger $C_{d}$ should tend to enhance this effect, as the magnitude of the frictional force increases relative to other forces acting on nearground parcels at the periphery of the vortex, in turn leading to increased radial inflow. Indeed, the tornado is characterized by a strong central updraft within the first one to three grid levels AGL in CD2-2 and CD5-2, but not in the weak-drag experiments; these differences are also evidenced in the time-height sections (Figs. 3a-e), which reveal a stronger updraft below $100 \mathrm{~m}$ AGL in CD2-2 and CD5-2 compared with the other experiments. This greater propensity for single-vortex structure over rough surfaces was also demonstrated in the tornado-like vortex laboratory experiments of Leslie (1977): a larger imposed swirl ratio was required to drive a transition from single- to multiple-vortex structure when the surface roughness was increased artificially in the laboratory chamber. Church et al. (1979) found comparable results in a separate laboratory experiment, concluding that "the swirl ratio is the internal parameter which primarily determines the [vortex] core configuration ... [but] the surface boundary layer plays 


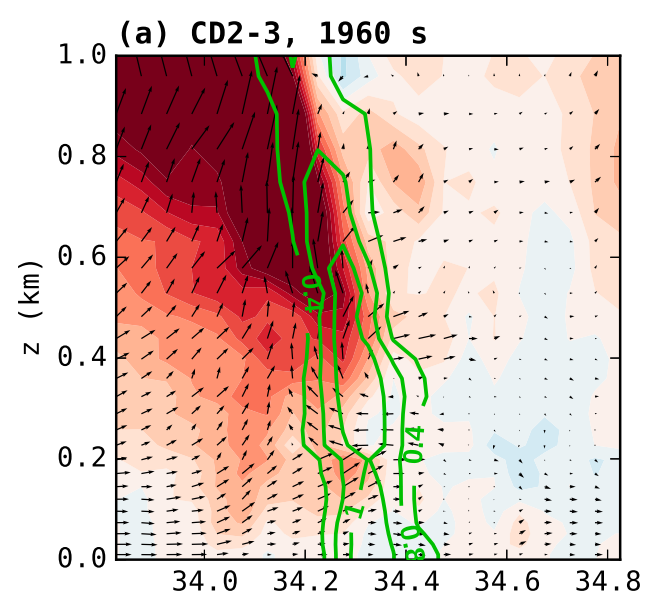

(b) $\mathrm{CD} 5-3,1780 \mathrm{~s}$
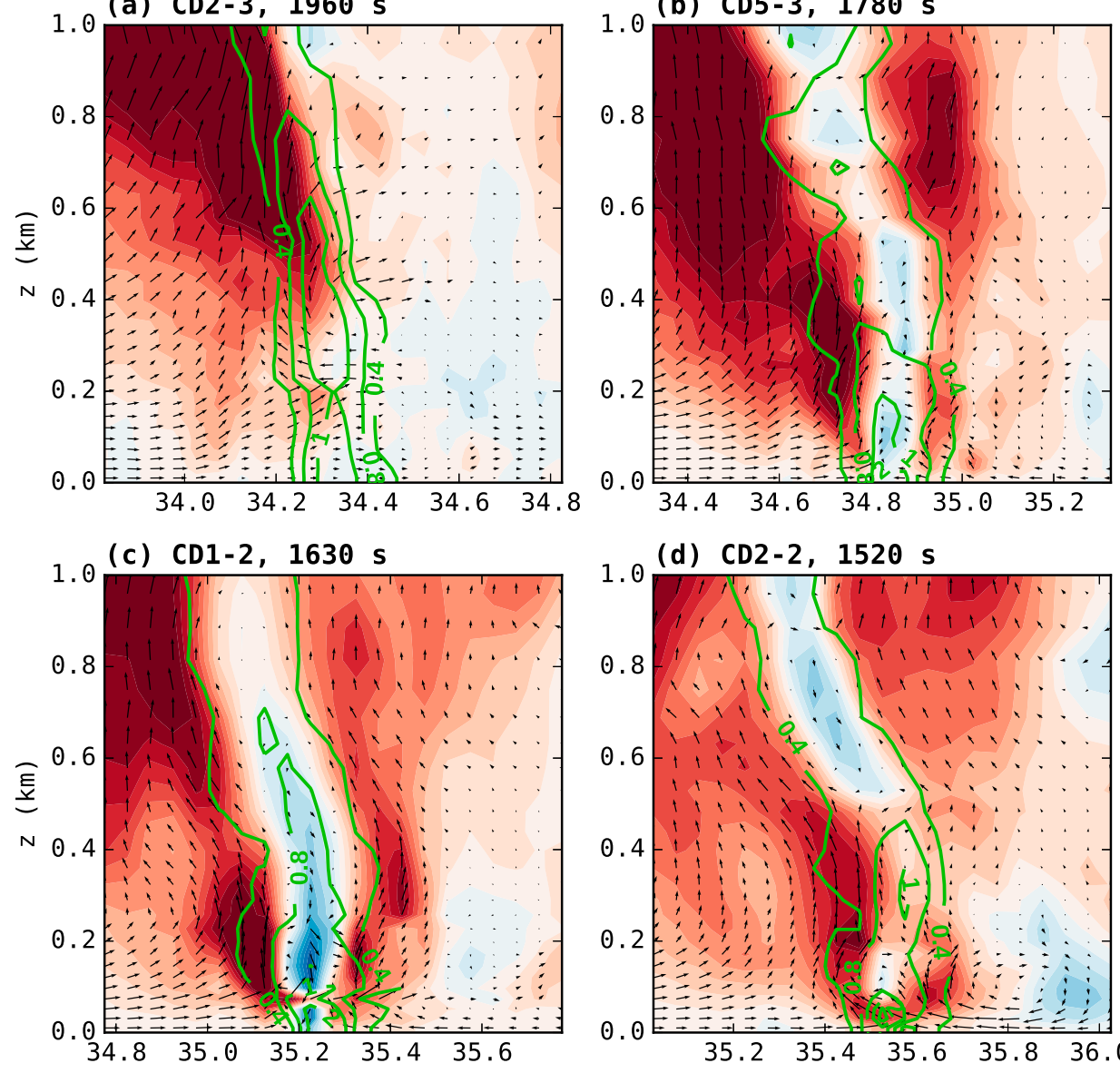

(d) $\mathrm{CD} 2-2,1520 \mathrm{~s}$
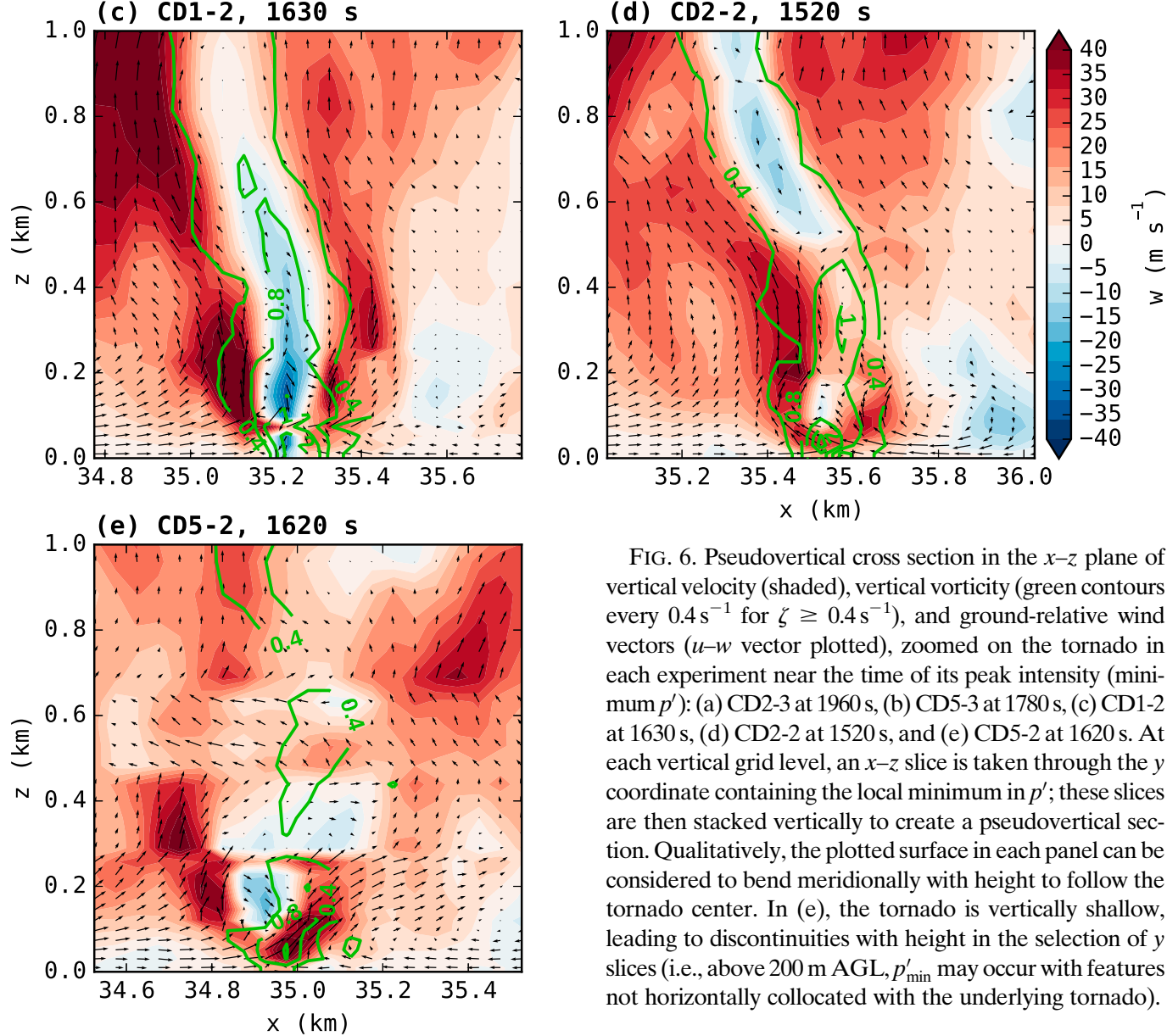

FIG. 6. Pseudovertical cross section in the $x-z$ plane of vertical velocity (shaded), vertical vorticity (green contours every $0.4 \mathrm{~s}^{-1}$ for $\zeta \geq 0.4 \mathrm{~s}^{-1}$ ), and ground-relative wind vectors $(u-w$ vector plotted), zoomed on the tornado in each experiment near the time of its peak intensity (minimum $p^{\prime}$ ): (a) CD2-3 at $1960 \mathrm{~s}$, (b) CD5-3 at $1780 \mathrm{~s}$, (c) CD1-2 at $1630 \mathrm{~s},(\mathrm{~d}) \mathrm{CD} 2-2$ at $1520 \mathrm{~s}$, and (e) CD5-2 at $1620 \mathrm{~s}$. At each vertical grid level, an $x-z$ slice is taken through the $y$ coordinate containing the local minimum in $p^{\prime}$; these slices are then stacked vertically to create a pseudovertical section. Qualitatively, the plotted surface in each panel can be considered to bend meridionally with height to follow the tornado center. In (e), the tornado is vertically shallow, leading to discontinuities with height in the selection of $y$ slices (i.e., above $200 \mathrm{~m} \mathrm{AGL}, p_{\min }^{\prime}$ may occur with features not horizontally collocated with the underlying tornado).

a significant yet secondary ... role." Finally, these results are also consistent with recent idealized numerical simulations of tornadoes interacting with changes in local surface roughness (Lewellen 2014).

More recently, Fiedler (2017, hereafter F17) conducted idealized experiments of an axisymmetric vortex with varying lower boundary conditions (including,

effectively, multiple drag coefficients for the semi-slip boundary condition) to predict how simulated tornadoes should behave in full $3 \mathrm{D}$ cloud models such as that used in the present study. F17 "anticipate[s] that a cloud model with $C_{d}=0.01 \ldots$ will produce tornadoes ... that would have properties close to being free-slip" in structure; specifically, downdraft would 

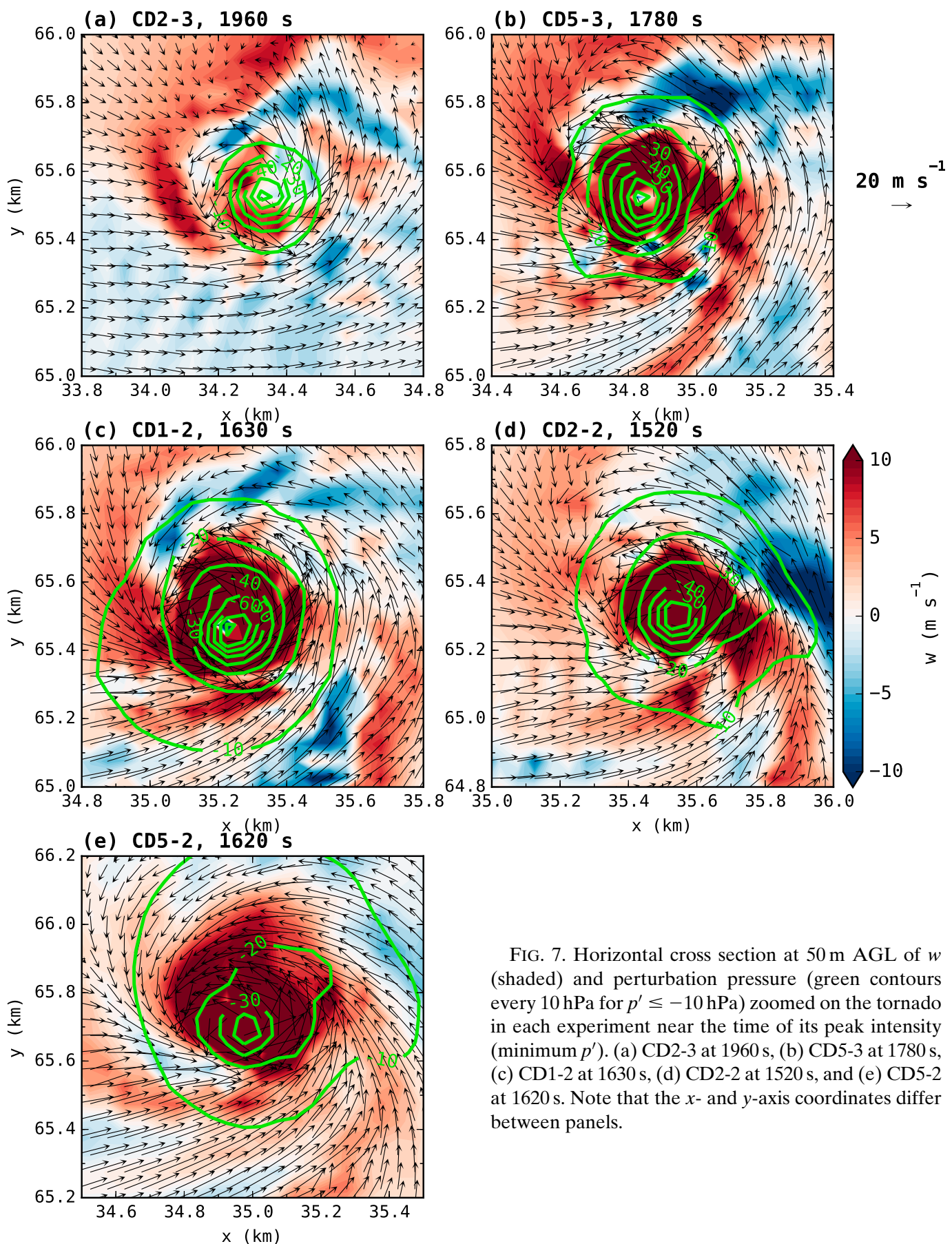

FIG. 7. Horizontal cross section at $50 \mathrm{~m}$ AGL of $w$ (shaded) and perturbation pressure (green contours every $10 \mathrm{hPa}$ for $p^{\prime} \leq-10 \mathrm{hPa}$ ) zoomed on the tornado in each experiment near the time of its peak intensity (minimum $p^{\prime}$ ). (a) CD2-3 at $1960 \mathrm{~s}$, (b) CD5-3 at $1780 \mathrm{~s}$, (c) CD1-2 at $1630 \mathrm{~s}$, (d) CD2-2 at $1520 \mathrm{~s}$, and (e) CD5-2 at $1620 \mathrm{~s}$. Note that the $x$ - and $y$-axis coordinates differ between panels.

be expected to penetrate down to ground level. Indeed, in CD1-2, strong downdraft exists in the vortex core near the ground at the time of peak intensity (Fig. 6c). Other experiments in F17 that effectively employed $C_{d}=0.0 \overline{3}$ and 0.1 showed the axial downdraft dislodged upward from the ground at most angular velocities tested (cf. their Figs. 3 and 4), matching the results herein for CD2-2 and CD5-2 (Figs. 6d,e).

Figure 8a presents vertical profiles of vertical mass flux through a $350 \mathrm{~m} \times 350 \mathrm{~m}$ horizontal box centered on the tornado in each experiment. The profiles represent $1 \mathrm{~min}$ averages ending at the time in each experiment when $p^{\prime}$ within the tornado reaches its minimum (cf. Fig. 2a). At each grid level and at each time, the box is centered on the grid point where $p^{\prime}$ is a local minimum. The upward mass flux increases monotonically with $C_{d}$ 


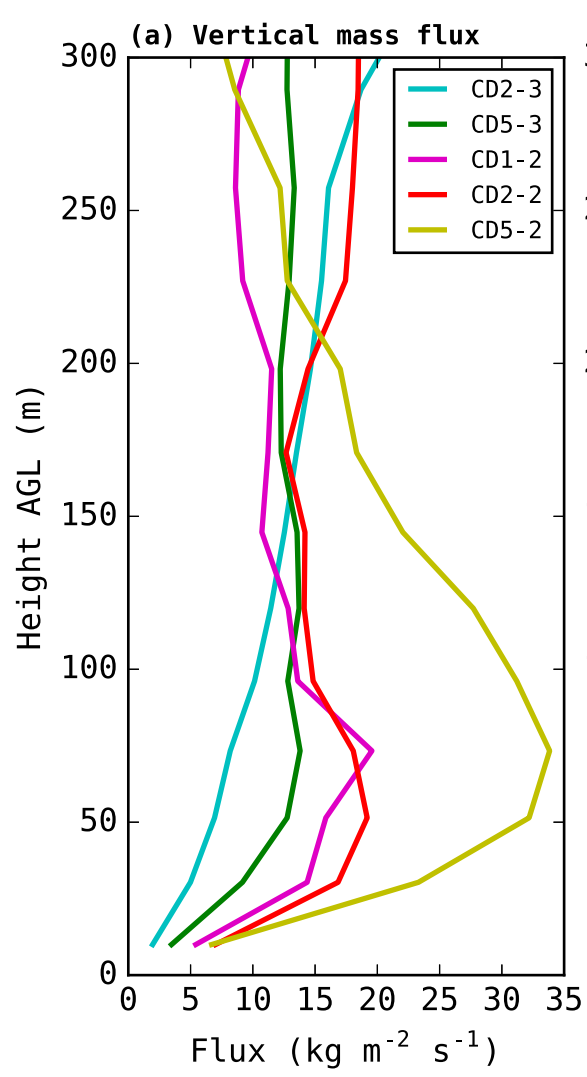

(b) Maximum w

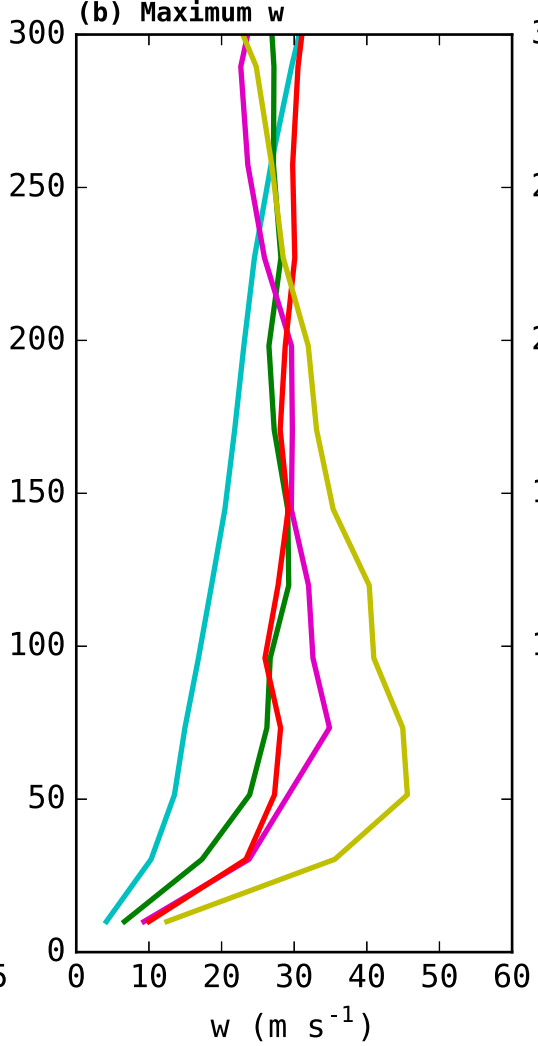

(c) Minimum w

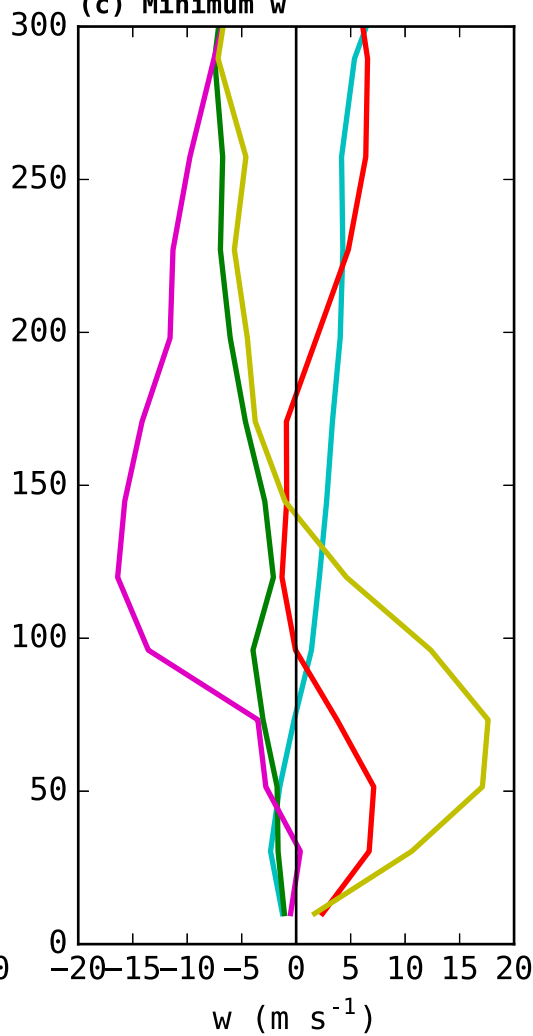

FIG. 8. Time-averaged vertical profiles of (a) vertical mass flux, (b) maximum $w$, and (c) minimum $w$ within a $350 \mathrm{~m} \times 350 \mathrm{~m}$ box centered on the first tornado in each experiment (except CD0, which has no tornado during the initial mesocyclone cycle). At each vertical level and at each sampled time, the box is centered on the grid point with minimum $p^{\prime}$. For each experiment, the tornado is sampled at $10 \mathrm{~s}$ intervals over the minute leading up to the time of the minimum $p^{\prime}$ in Fig. 2 (1900-1960 s for CD2-3; 1720-1780 s for C5-3; 1570-1630 s for CD1-2; 1460-1520 s for CD2-2; 1560-1620 s for CD5-2).

within the lowest $70 \mathrm{~m} \mathrm{AGL}$, despite the fact that overall tornado intensity does not exhibit this monotonic increase (indeed, well away from the ground at $300 \mathrm{~m}$ AGL, the largest upward flux is actually found in the weakest-drag experiment). A similar trend is found for the time-average maximum $w$ inside the $350 \mathrm{~m} \times 350 \mathrm{~m}$ box (Fig. 8b). Furthermore, when examining timeaverage minimum $w$ inside the box, evidence of axial downdraft penetrating down to the lowest $50 \mathrm{~m}$ AGL is most prevalent in CD2-3 and CD5-3; modest downdraft also occurs above $30 \mathrm{~m}$ AGL in CD1-2. By contrast, downdraft is entirely absent below $100 \mathrm{~m}$ AGL in CD2-2 and CD5-2. These results mirror aspects of Nolan et al. (2017), who showed in very high-resolution idealized 3D LES tornado simulations that the maximum updraft speed at $10 \mathrm{~m}$ AGL in their vortices increased markedly with surface roughness (among three experiments with $z_{0}=0.05,0.2$, and $0.8 \mathrm{~m}$; cf. their Table 3 ). To the extent that our time-averaged profiles in Fig. 8 represent the overall tornado behavior in each experiment, our results support the arguments of T00 and confirm that high-resolution storm-scale numerical simulations can reproduce certain aspects of tornadic structure previously identified in highly idealized vortex models with artificial forcing.

\section{c. Circulation analysis of early mesocyclone}

To examine the dynamics of mesocyclone intensification, material circuits will once again be employed, as in RX17. In this case, it is of particular interest to determine whether the contribution to mesocyclone circulation from surface drag increases in an orderly fashion as $C_{d}$ increases. The procedure for initializing the circuits, as well as for calculating circulation and its forcing terms along the circuit, is the same as in RX17 in most respects; a brief review follows here. Horizontal circular circuits of radius $1.5 \mathrm{~km}$ are initialized centered on the low-level mesocyclone (determined subjectively from the model wind field) with parcels approximately $19 \mathrm{~m}$ apart. These parcels are integrated backward in time as trajectories; when the distance between adjacent parcels exceeds $25 \mathrm{~m}$ after an integration time step, a 
new parcel is added to the circuit at the midpoint of the line segment connecting those parcels, and is then included at all subsequent (backward) time steps. We integrate circuits backward in time for $600 \mathrm{~s}(10 \mathrm{~min})$ at a time step of $0.5 \mathrm{~s}$ (afforded by linear temporal interpolation of the wind between model data files, which are available every $2 \mathrm{~s}$ ). After integration, the relevant state variables are interpolated to parcel locations in order to compute the circuit's circulation and circulation forcing terms at each model data time (every $2 \mathrm{~s}$ ). One notable difference from RX17 is that the GWB technique, which applies a PPGF and the Coriolis force, introduces new terms into the prognostic circulation equation for a circuit such that

$$
\frac{d C}{d t}=\oint \mathbf{F} \cdot d \mathbf{l}+\oint B d z+\oint \mathbf{P} \cdot d \mathbf{l}-\oint(2 \mathbf{\Omega} \times \mathbf{v}) \cdot d \mathbf{l},
$$

where $\mathbf{F}$ is the total mixing force, $B$ is buoyancy, $\mathbf{P}$ is the PPGF (as specified by the GWB technique), $\boldsymbol{\Omega}$ is Earth's rotation, $\mathbf{v}$ is the velocity vector, $d \mathbf{l}$ is a circuit segment (directed counterclockwise), and $d z$ is the vertical component of the segment. From left to right, the RHS terms in (1) represent circulation forcing from mixing, baroclinity, the PPGF, and Coriolis. ${ }^{5}$ Note that $\mathbf{F}$ represents the net action of subgrid-scale turbulence and numerical diffusion on the velocity components; when a parcel is near the ground and $C_{d}>0$, the effects of surface drag typically dominate this term.

In the present study, we initialize circuits in each experiment across an array of initial heights and times. For each experiment, we initialize a circuit at three heights $(500,1000$, and $2000 \mathrm{~m}$ AGL) at four times $(1200,1260$, $1320,1380 \mathrm{~s}$ ); this yields 12 total circuits per experiment. Our goal is to track how the forcing terms affect the lowlevel mesocyclone circulation during its period of initial intensification. Dahl et al. (2012) discussed the increased uncertainty associated with trajectories in 3D numerical simulations on the C grid (Arakawa and Lamb 1977) that pass below the lowest scalar grid level AGL. While it is often possible to select trajectories for which this conundrum does not apply when analyzing individual parcels (e.g., R16), it is impractical to do so for a large material circuit integrated over a duration of $10 \mathrm{~min}$, considering the number of parcels entailed. Consequently, as in RX17, we accept that some constituent parcels will pass below $10 \mathrm{~m}$ AGL (the height of our first scalar level); in such cases, all interpolated scalar quantities and horizontal momentum components

\footnotetext{
${ }^{5}$ In our simulations, Coriolis is calculated with the domain's center latitude taken to be $36^{\circ} \mathrm{N}$.
}

are taken to be their values directly above the parcel at $10 \mathrm{~m}$ AGL (note that $w$ and its forcing terms are defined on the $\mathrm{C}$ grid at the lower boundary, obviating the need for this special treatment). This treatment avoids extrapolation, but we still expect increased errors in both the trajectory position and interpolated quantities (e.g., for our circulation budgets) when it is applied. An analysis of the height distribution for all parcels comprising our circuits (not shown) revealed that, at any given time, no more than $12 \%$ of parcels resided below $10 \mathrm{~m}$ AGL for any circuit; a more typical proportion during the early part of the integration windows was $5 \%$. In practice, we expect analysis of these circuits to yield qualitatively valid results when their interpolated (i.e., model predicted) circulation agrees reasonably well with the circulation integrated from source terms throughout the budget integration period.

Figure 9 presents bar charts showing the integrated circulation contributions from the mixing (Figs. 9a-c) and baroclinic (Figs. 9d-f) forcing terms over the preceding $10 \mathrm{~min}$, normalized by the circuit's final circulation value ${ }^{6}$ (the PPGF and Coriolis forcing terms are omitted for clarity here, as the former is an artifact of our simulation approach and the latter is typically too small to be of interest in supercell dynamics). It should be emphasized that each initial circuit time labeled on the abscissa represents a unique set of circuits (e.g., the four yellow bars in Fig. 9a represent the normalized mixing contribution for four unique circuits in CD5-2, not the time evolution of a single circuit in CD5-2). Nonetheless, within a given experiment and at a given initial height, we take the series of four circuits initialized at 60 s intervals between 1200 and 1380 s to represent the time evolution of certain bulk mesocyclone properties-in particular, the proportion of mesocyclone circulation generated by mixing and baroclinity.

For circuits initialized at $500 \mathrm{~m}$ AGL, the mixing term imparts a net negative contribution of $15 \%-30 \%$ of the mesocyclone's circulation for each of the initialization times in experiments $\mathrm{CD} 0$ and $\mathrm{CD} 2-3$; the relative magnitudes of these contributions generally remain steady with time over the period (Fig. 9a). By contrast, the mesocyclone in CD5-3, CD1-2, CD2-2, and CD5-2 sees an increased contribution with time from the mixing term during the same period. For CD5-3, the contribution at $1200 \mathrm{~s}$ is weakly negative, but becomes

\footnotetext{
${ }^{6}$ The quantities plotted are given as the ratio of the circulation generated by the forcing term during the 10 min integration period to the instantaneous value of the circuit's circulation at the end of the integration period; this ratio is expressed as a percentage for clarity.
} 

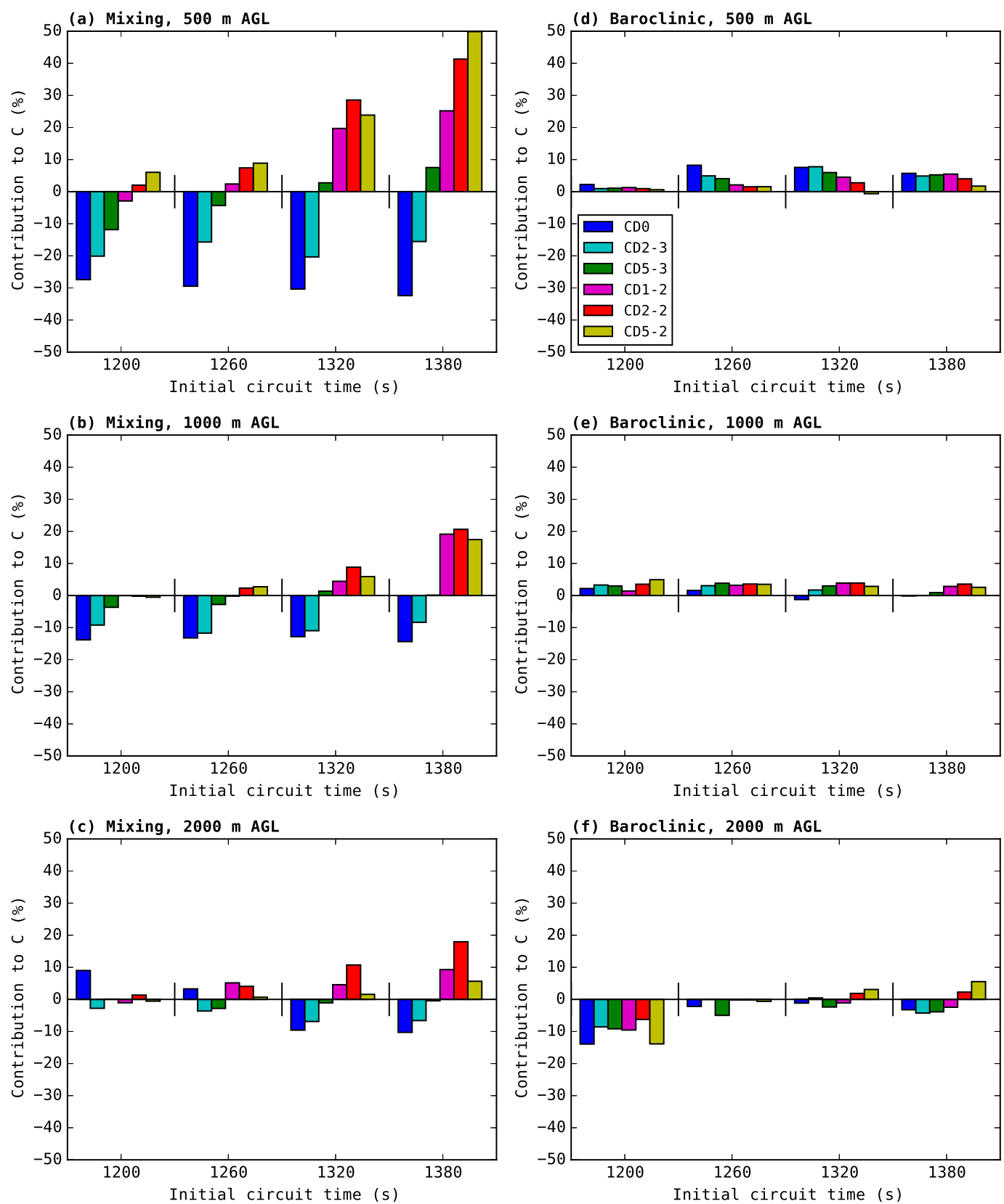

FIG. 9. Total contribution by the mixing generation term over the $10 \mathrm{~min}$ circulation budget integration window, normalized by the final value of circulation at the end of the window, for circuits initialized at (a) 500, (b) 1000, and (c) $2000 \mathrm{~m}$ AGL. (d)-(f) As in (a)-(c), but for the baroclinic generation term. Each panel is divided into four sections corresponding to the times labeled on the abscissa. These labels denote when the circuit in each experiment was initialized; the plotted contributions occurred over the $10 \mathrm{~min}$ period preceding this time.

weakly positive by $1380 \mathrm{~s}$. For CD2-2 and CD5-2, the mixing contribution at $1200 \mathrm{~s}$ is small but positive, but grows increasingly large with time; by $1380 \mathrm{~s}$, mixing generation accounts for $40 \%-50 \%$ of the circuits' circulation. In all experiments and at all times, the contribution from baroclinity is small, accounting for no more than $10 \%$ (negative or positive) of the final circulation
(Fig. 9d). We believe the increase in the relative contribution of mixing with time in CD5-3, CD1-2, CD2-2, and CD5-2 owes to the same positive feedback process described at length in RX17 (for experiment FWFRIC therein): as the low-level mesocyclone begins to intensify, the coincident low-level updraft strengthens dynamically, allowing more frictional vorticity residing in 
the lowest few hundred meters AGL to be ingested into the circulation; this, in turn, enhances the mesocyclone in a positive feedback loop.

For the circuits initialized at $1000 \mathrm{~m}$ AGL, a similar trend in the mixing-term contribution with time and with $C_{d}$ is seen (Fig. 9b), albeit with smaller relative magnitudes than for the circuits at $500 \mathrm{~m}$ AGL. One notable difference at $1000 \mathrm{~m}$ AGL is that, by the time of rapid mesocyclone intensification at $1380 \mathrm{~s}$, mixing is actually more effective at generating positive circulation in CD2-2 than in CD5-2. Once again, as was true at $500 \mathrm{~m}$ AGL, baroclinity plays only a small role in generating circulation for the mesocyclone at $1000 \mathrm{~m}$ AGL (Fig. 9e). Finally, at $2000 \mathrm{~m}$ AGL, the contribution of the mixing term is smaller still in magnitude and less correlated with time or $C_{d}$ (Fig. 9c) than at lower heights. Except for CD2-2, where mixing is a $10 \%-20 \%$ positive contribution by 1320 and $1380 \mathrm{~s}$, mixing contributions for other circuits are small and on the same order of magnitude as baroclinity (Fig. 9f). We note that while the circuits initialized at $2000 \mathrm{~m}$ AGL contain only a small relative contribution from frictional generation, it is possible that more substantial such generation occurred earlier in the simulation than our $10 \mathrm{~min}$ integration window (e.g., if constituent parcels were located near the ground $>10 \mathrm{~min}$ before our circuit initialization time but ascended thereafter, our integration window would potentially miss important frictional generation).

The trend for the mixing term to provide a more positive contribution to the low-level mesocyclone circulation as $C_{d}$ increases is expected, based on the mechanism identified in RX17 wherein surface drag slows the southwestward-directed momentum of nearground inflow parcels while parcels higher above are less affected by the surface drag. To better understand the physical mechanisms responsible for the mixing contributions shown in Fig. 9a, three-dimensional circuits are plotted in Fig. 10, with each interparcel segment shaded by its local per-unit-length contribution to the mixing term. While the circuits plotted were initialized around the mesocyclone at $1380 \mathrm{~s}$ and $500 \mathrm{~m}$ AGL in each experiment, their positions are plotted at $1140 \mathrm{~s}(4 \mathrm{~min}$ into their backward integration). As seen in circulation budgets for these circuits (Fig. 11), the magnitude of the mixing term tends to be maximized around this time (1140s), regardless of whether its sign is predominantly positive (CD5-3, CD1-2, CD2-2, and CD5-2) or negative (CD0 and CD2-3) during the integration window. It is apparent in Fig. 10 that the circuits in all six experiments contain a long segment lying near the ground toward their southeastern extent, similar to circuits previously analyzed in EnvFRIC and FWFRIC (cf. Fig. 9 in RX17). Along most of this near-ground segment, which lies in the inflow region east of the mesocyclone, the sign of the local mixing term reflects the predominant sign seen in Figs. $9 \mathrm{a}$ and 11 for the total circuit generation term. In all experiments, some locally large values of this generation term are seen along higher portions of the circuit toward its northwestern extent, but these tend to manifest as offsetting dipoles with opposite signs on the upwardand downward-directed circuit segments. Thus, the long near-ground circuit segment in the inflow region appears primarily responsible for the net forcing from mixing in each experiment, implicating the effects of surface drag (or lack thereof in CD0). These budgets further corroborate the conceptual model of the frictional generation mechanism from RX17 (e.g., their Fig. 15) and verify its presence over the $C_{d}$ parameter space we examine herein: in CD5-3, CD1-2, CD2-2, and CD5-2, surface drag is acting against northeasterly ${ }^{7}$ near-ground flow in the inflow region. This decelerates flow that is locally consistent with clockwise (negative) circulation about the circuit, thus increasing the total circulation. The same mechanism acts on the circuits at $1000 \mathrm{~m}$ AGL, but it constitutes a relatively smaller portion of the final circulation (Fig. 9b), because the circuit is farther away from ground and thus less affected by surface drag. At $2000 \mathrm{~m}$ AGL, mixing has only a modest impact on circulation overall (Fig. 9c).

A noteworthy result is that, in the absence of drag, mixing imposes a substantial negative contribution to the final circulation at $500-1000 \mathrm{~m}$ AGL in CD0; this is also true to a lesser extent in CD2-3 with weak drag. As discussed in Markowski (2016) (see their Fig. 24) and supported in RX17, internal mixing typically acts to dampen local vorticity maxima (e.g., the large horizontal vorticity in the inflow region east of the mesocyclone). The circulation budgets at $500 \mathrm{~m}$ AGL for CD0 suggest this effect can act to impart a negative contribution of as much as $25 \%-30 \%$ to the circuits' circulation during their approach to the low-level mesocyclone (e.g., Fig. 9a). This provides a baseline that puts the mixing contribution for the other experiments into context: in experiments CD2-2 and CD5-2, where the mixing term provides a $40 \%-50 \%$ positive net contribution to circulation, the final circulation is perhaps as much as $150 \%$ larger than might be expected in the absence of drag. ${ }^{8}$

\footnotetext{
${ }^{7}$ Here we refer to northeasterly flow in a ground-relative sense, as seen in Fig. 4.

${ }^{8}$ This assumes 1) the same initial circuit position and subsequent trajectory, 2) the same initial circulation at the beginning of the integration window, 3) similarly negligible contributions from PPGF and Coriolis, and 4) that diffusive effects alone would impart the same $25 \%-30 \%$ negative contribution during the integration window as seen in CD0.
} 
(a) $\operatorname{CDO}$

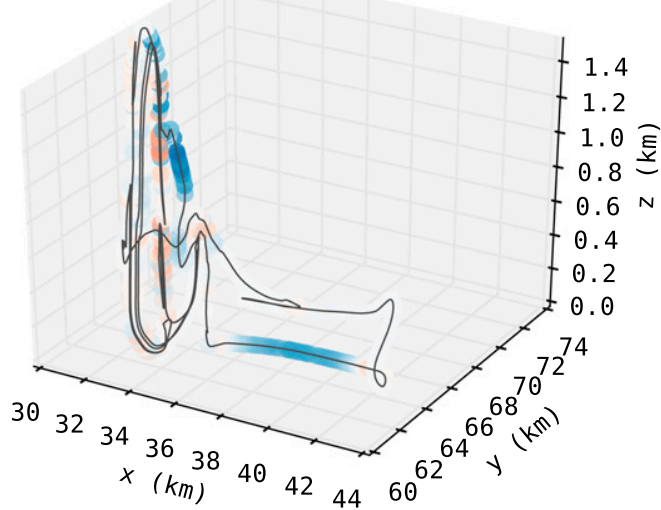

(c) $\mathrm{CD} 5-3$

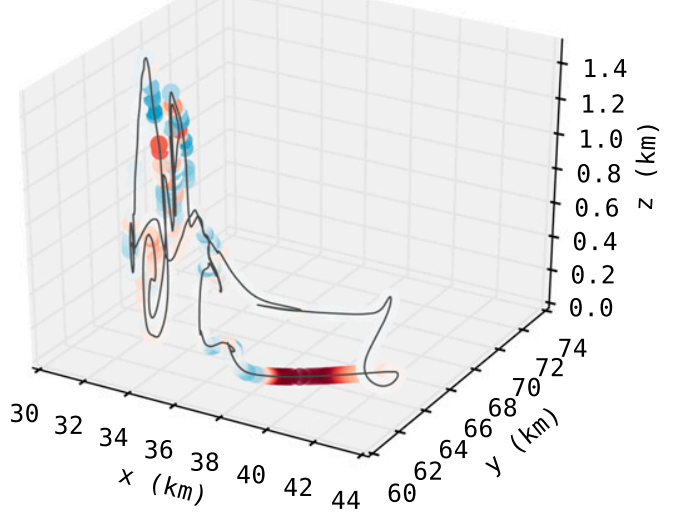

(e) $\mathrm{CD} 2-2$

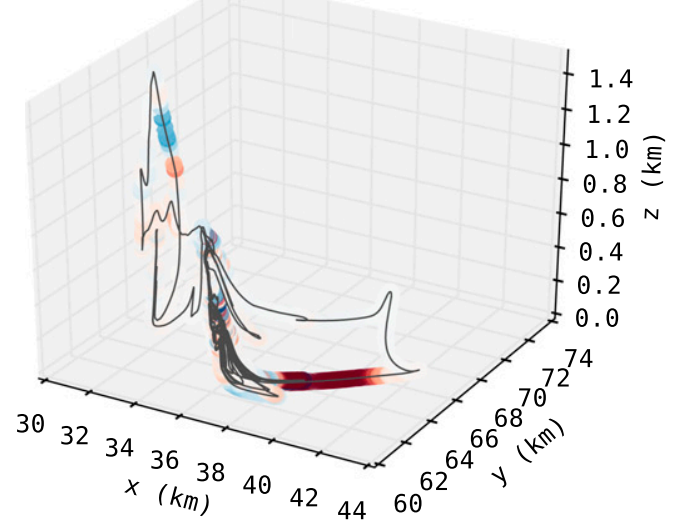

(b) $\mathrm{CD} 2-3$

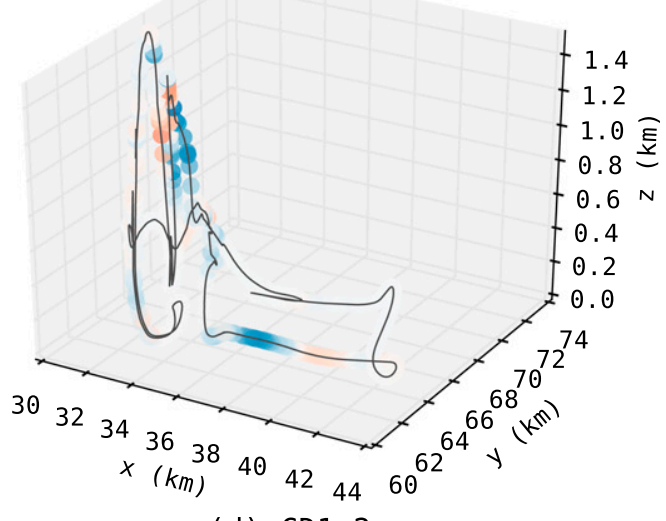

(d) CD1-2

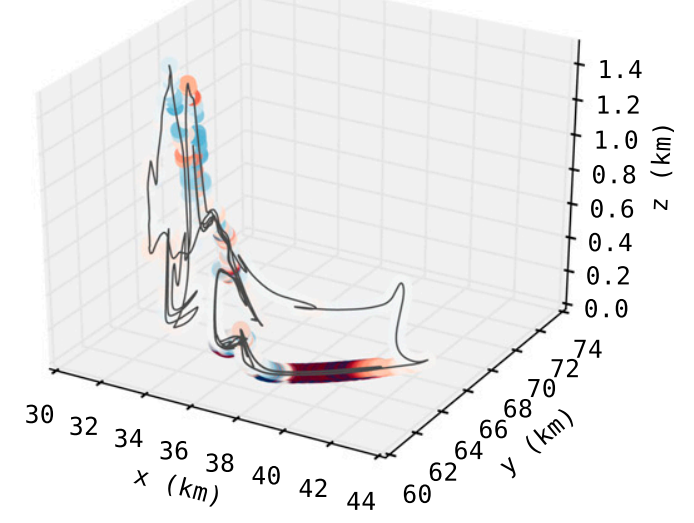

(f) $\mathrm{CD} 5-2$

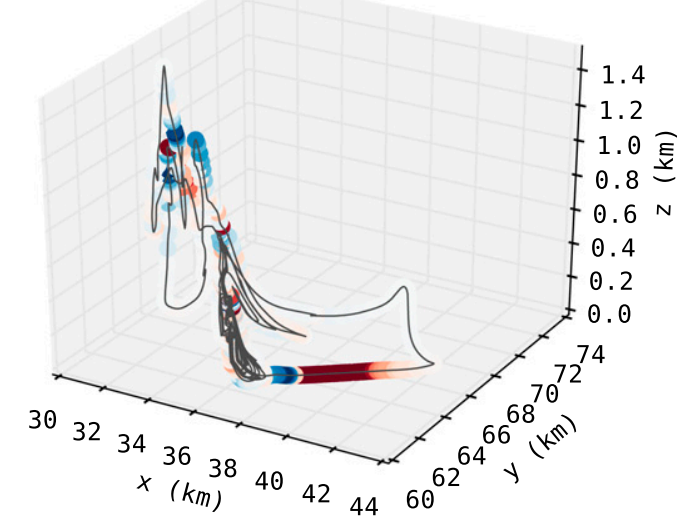

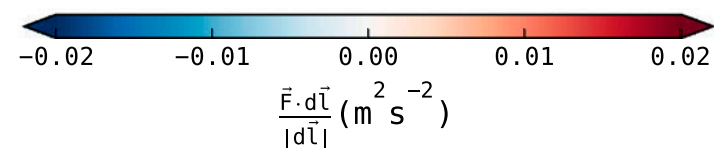

FIG. 10. For circuits initialized at $1380 \mathrm{~s}$ and $500 \mathrm{~m}$ AGL, the circuit position at $1140 \mathrm{~s}$ is plotted for the circuit in (a) CD0, (b) CD2-3, (c) CD5-3, (d) CD1-2, (e) CD2-2, and (f) CD5-2. Parcels are colored by $\mathbf{F} \cdot d \mathbf{l} /|d \mathbf{l}|$ (the "mixing term") for the adjacent circuit segment, which represents the local contribution to $\mathbf{F} \cdot d \mathbf{l}$ for that segment. 

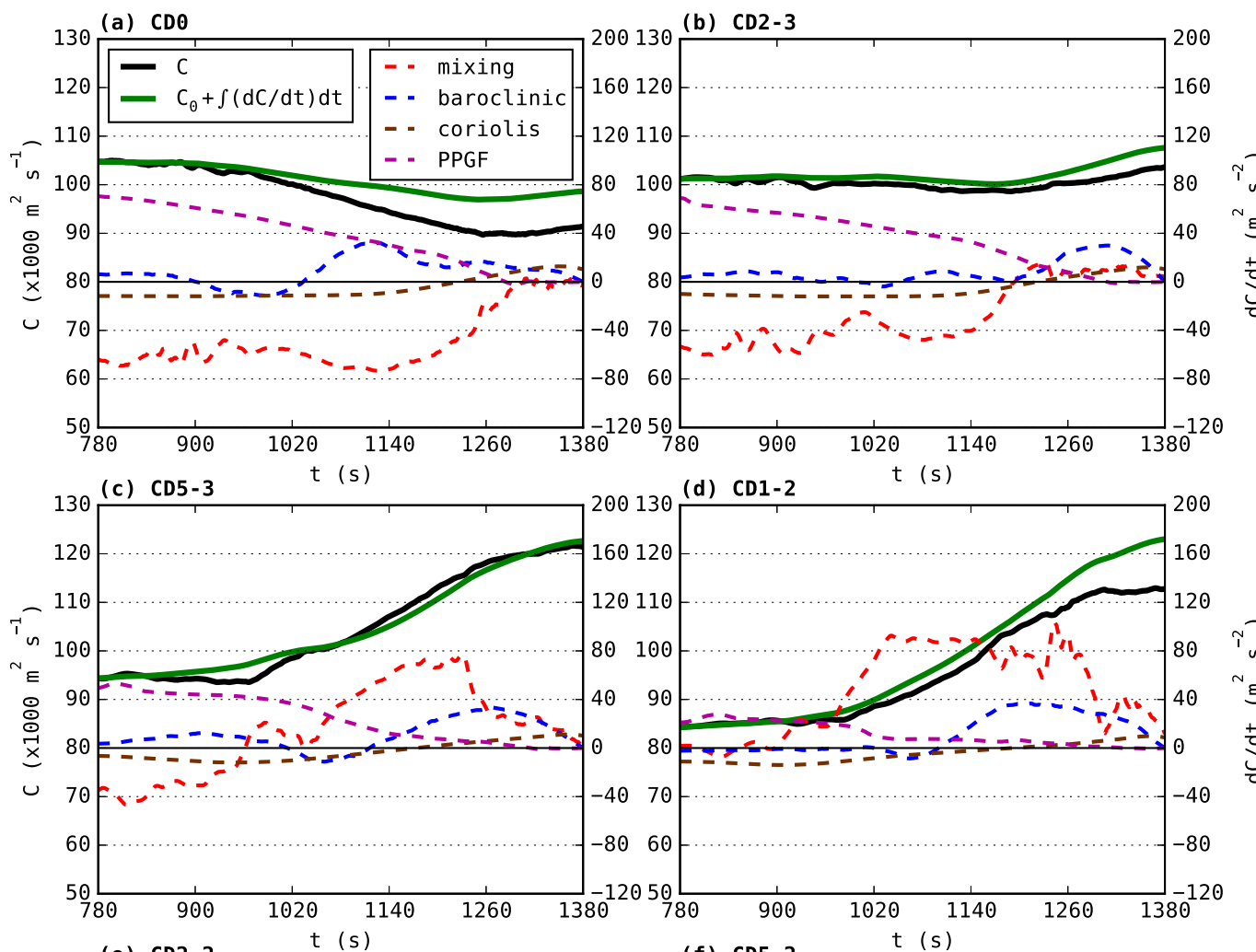

(d) CD1-2
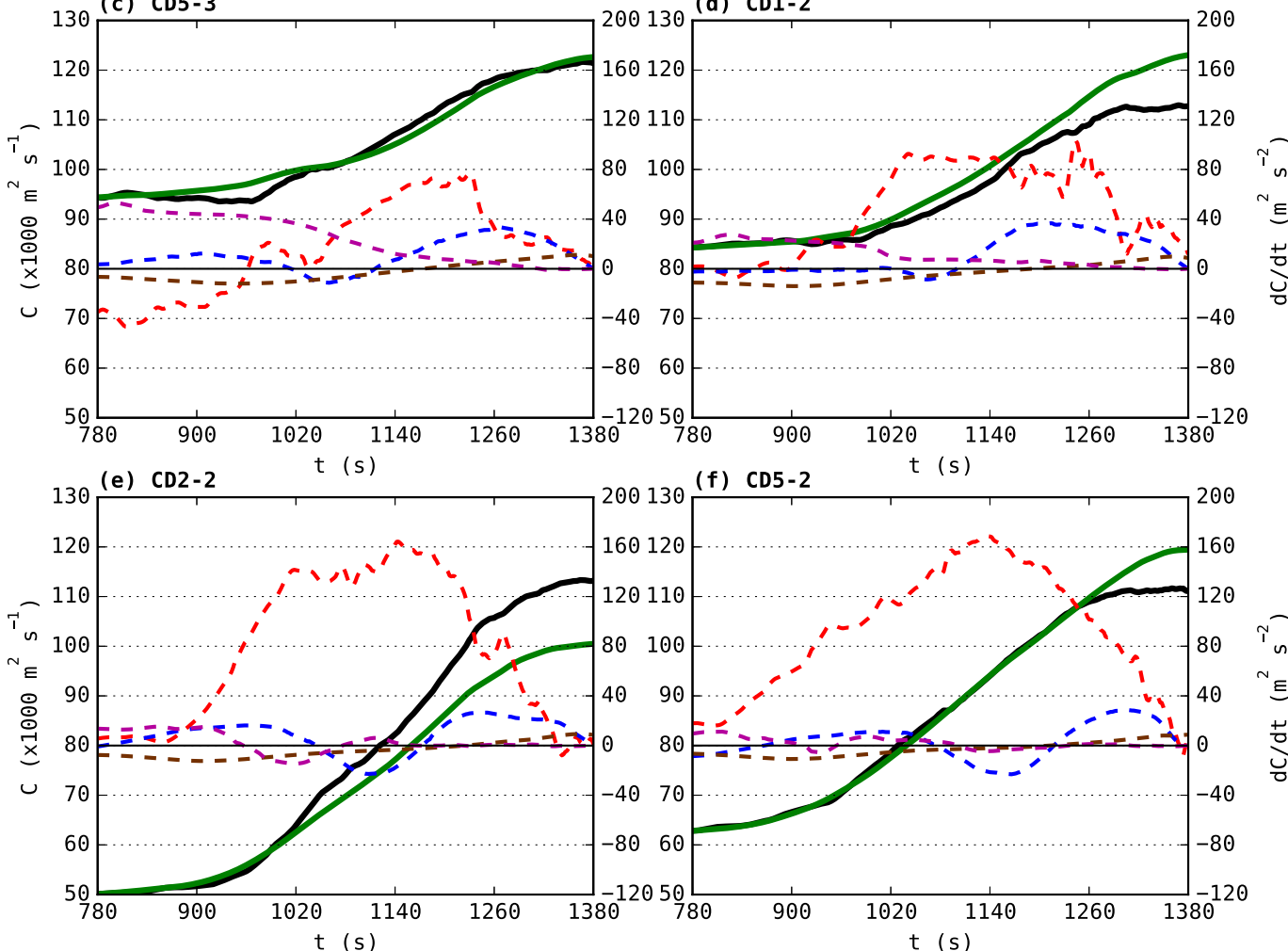

FIG. 11. Time series of interpolated circulation (solid black) and circulation integrated from forcing terms (solid green), and for the mixing (dashed red), baroclinic (dashed blue), Coriolis (dashed brown), and PPGF (dashed purple) forcing terms for the circuits in Fig. 10. panels here correspond to the circuits described therein. These circuits were each initialized at $1380 \mathrm{~s}$ and integrated backward $10 \mathrm{~min}$ in time (to $780 \mathrm{~s}$ ). The left ordinate axis labels are for the interpolated and integrated circulation, while the right ordinate axis labels are for the forcing terms.

Even in CD5-3, where mixing has just a small positive net contribution to the mesocyclone circulation at $1380 \mathrm{~s}$, surface drag itself is likely still generating substantial circulation (e.g., Fig. 10c) that is mostly offset by the diffusive effects of internal mixing. One caveat to interpreting the mixing forcing in $\mathrm{CD} 0$ as a "baseline" for the other experiments is that agreement between its circuit's interpolated and integrated circulation budgets is only modest (Fig. 11a). Note that some disagreement between interpolated and integrated circulation is unavoidable due to numerical errors often related to near-grid-scale features.

\section{Summary and conclusions}

In this study, a new method (DRX19) was employed for maintaining a three-force balance among the horizontal PGF, Coriolis force, and frictional force in the background environment of idealized single-sounding 3D storm simulations. This geotriptic wind balance 
(GWB) technique allows the use of an arbitrary initial sounding in simulations that use parameterized surface drag with constant drag coefficient $C_{d}$; without the GWB, surface drag would act to modify the background wind profile over time throughout the domain, particularly near the ground. The GWB technique was employed in six idealized supercell simulations whose drag coefficients spanned the range $0 \leq C_{d} \leq 0.05$. All the simulations with nonzero drag coefficients produced a low-level mesocyclone 1200-1800s into the simulation that lowered toward the ground and eventually spawned a strong tornado, similar to experiment FWFRIC in RX17. The experiment with $C_{d}=0$ was very similar to EnvFRIC in RX17, and did not produce a tornado nor an intense near-ground mesocyclone during this period.

Material circuits were initialized enclosing the lowlevel mesocyclone during its early intensification phase, integrated backward in time, and circulation budgets were calculated. These budgets suggest surface drag contributed a larger positive proportion of the total circulation for circuits in the experiments with larger drag coefficients during this early mesocyclone intensification period. Furthermore, the budgets for circuits in CD0 reveal that in the absence of surface drag, mixing processes (turbulence mixing and numerical diffusion) commonly imposed a substantial (15\%-25\% below $1 \mathrm{~km}$ AGL) negative contribution to circulation on circuits bound for the low-level mesocyclone (note that the surface drag effect is propagated into the interior flow from the ground surface through the turbulence mixing terms in the numerical model). Thus, the positive net contribution from mixing seen in the strong-drag experiments suggests the beneficial effect of surface drag was large enough to overcome a baseline negative contribution from other mixing effects.

Additionally, vertical cross sections through the tornadoes (in experiments that produced them) revealed structure consistent in some respects with previous laboratory experiments (Ward 1972) and numerical simulations using axisymmetric models with surface drag (T00; F17). Specifically, radial inflow along the ground toward the center of tornadoes in the strongdrag experiments was substantially stronger than those in the weak-drag and no-drag experiments. Also, an axial downdraft in the tornadoes penetrated down to the first grid level AGL in the experiments with smaller $C_{d}$; in the two strongest-drag experiments, however, this downdraft was dislodged upward at least two grid levels AGL.

Following R16 and RX17, the experiments in the present study strengthen some of our earlier key findings regarding mesocyclone and tornado behavior in the presence or absence of surface drag. In the most important respects (e.g., timing of intensification and lowering toward the ground), the low-level mesocyclone behavior changed monotonically and fairly predictably with $C_{d}$ over the range of values tested herein, up until the strongest-drag experiment (CD5-2) with a drag coefficient of 0.05 . Given the decreased intensity of the tornado and low-level updraft in CD5-2 relative to CD2-2, we infer that for the bulk drag parameterization used in most current atmospheric models, the optimal value of $C_{d}$ for generating an intense near-ground mesocyclone in conditions similar to ours lies between 0.01 and 0.05 . Although $C_{d}$ was spatially homogeneous in our simulations, given the circulation analyses presented in Fig. 10, it is likely that the drag strength in the inflow region of the supercell was the dominant control on generation of important circulation [although in the general case, this same effect could occur in other regions of the storm (e.g., the RFD), provided parcels originating there subsequently enter the tornado]. This broadly agrees with some aspects of Reames and Stensrud (2018, hereafter RS18), who produced a 108 -member ensemble of $500 \mathrm{~m}$ real-data simulations based on the 31 May 2013 El Reno, Oklahoma, supercell; in each member, land surface properties corresponding to the Dallas-Fort Worth urban area were specified over a different patch of the domain, with the remainder of the $250 \mathrm{~km} \times 250 \mathrm{~km}$ domain composed of grassland. RS18 found typically on the order of a $50 \%$ surplus in $0-1 \mathrm{~km}$ storm-relative helicity over the urban area, and ensemble members with the urban area placed south and southeast of the simulated storm track had a particular tendency toward a more intense second mesocyclone cycle (after storm maturity) than other members. The simulations in RS18 used real (heterogeneous) data for their initial condition, were much coarser than ours in horizontal resolution, and employed a PBL parameterization for boundary layer mixing instead of threedimensional subgrid-scale turbulence mixing as in our LES-type simulations; their results thus provide somewhat independent support for the notion that land surfaces beneath or near a supercell's inflow region with strong drag may enhance mesocyclone intensity. To the extent this notion is valid, it could provide a crucial opportunity for operational meteorologists to consider land surface properties in anticipating supercell behavior on short time scales. However, the relevance of these results to the real world, and even to numerical simulations across a broad range of environmental conditions, is subject to further investigation. For example, Markowski and Bryan (2016, hereafter MB16) illustrated the potential for overestimation of near-surface shear in LES when the modeled flow does not contain resolved eddies, an 
issue that may have some relevance to the simulations in the present study. ${ }^{9}$ Most recently, Markowski et al. (2019, hereafter M19) have discussed reasons for caution in applying conventional formulations of the "semi-slip" lower boundary condition in severe storm simulations, owing in part to field observations of larger vertical shear in the surface layer than is assumed in these formulations. However, M19 note that while field observations suggest the near-ground shear in typical storm outflow may severely violate those assumptions, violations in the inflow region and background environment are usually milder. With this considered, we believe that our results are at least qualitatively correct, especially in terms of the trend of surface drag dependency; using a more sophisticated (but currently unavailable) drag parameterization would most likely yield results with similar trends and key mechanisms (although storm and vortex behavior at particular values of $C_{d}$ is perhaps likely to change).

The results presented in this study constitute a step forward toward understanding surface drag's role in supercell tornadogenesis dynamics, but many steps remain. One such step is to perform experiments similar to those presented herein for a range of different initial soundings, which should help to illuminate which of our results are generalizable to most storms. Another step is to decrease the horizontal grid spacing by a factor of 2 or 3 to better resolve tornadoes; cross sections presented herein showed indications that our grid is just fine enough to simulate some semblance of multiple-vortex structure (e.g., Fig. 6), but that the subvortices are only marginally resolved, yielding unrealistic details. Adding more vertical grid levels within the lowest $100 \mathrm{~m}$ AGL could also prove immensely helpful in calculating vorticity and circulation budgets along trajectories and circuits bound for tornadoes and low-level mesocyclones, as we have found such parcels tend to originate from below $10 \mathrm{~m}$ AGL (our lowest scalar level) quite often. The higher vertical resolution near the surface can also better resolve vertical wind shear there and may make the results less sensitive to the surface-layer drag parameterization. The simulations herein also still contain mostly laminar flow in the inflow region, which could be subject to developing exaggerated near-ground

\footnotetext{
${ }^{9}$ A constant wind profile with height was specified in the initial conditions of MB16's idealized experiments, thus requiring an Ekman layer to develop from scratch during their simulations. It is therefore likely that the overestimation of near-wall shear demonstrated in MB16 was more severe than in our present study, given that we initialize with a realistic wind profile that has already been subject to the effects of surface drag, and we do not require the model to create a boundary layer.
}

shear as described by MB16. Thus, it may be desirable to eliminate this caveat by inducing turbulence in the far field with small thermal perturbations (Muñoz-Esparza et al. 2014; MB16; DRX19). Perhaps most crucially of all, borrowing more sophisticated surface-layer parameterizations from the engineering community (as suggested by M19) is a challenging but necessary step toward bolstering confidence that our storm simulations are reflecting the influence of drag realistically. If these considerations are addressed in the course of designing future idealized supercell simulations, then alongside real-data modeling studies and observational efforts, we are optimistic that an important component of the tornadogenesis problem - the relative importance of surface drag in generating tornadic vorticity across the full distribution of real-world tornado cases-may soon come into clearer focus.

Acknowledgments. This work was supported by NSF Grants AGS-1261776 and AGS-1917701, NOAA VORTEX-SE Grant NA17OAR4590188, and NSFC Grant 41730965. Numerical simulations were performed at the Texas Advanced Supercomputing Center, an NSF XSEDE facility. Portions of this work previously appeared in the Ph.D. dissertation of the first author (Roberts 2017). The authors wish to thank four anonymous reviewers for insightful critiques of the manuscript, which led to substantial improvements and additions.

\section{REFERENCES}

Arakawa, A., and V. R. Lamb, 1977: Computational design of the basic dynamical processes of the UCLA general circulation model. General Circulation Models of the Atmosphere, J. Chang, Ed., Methods in Computational Physics: Advances in Research and Applications, Vol. 17, Academic Press, 173265.

Bluestein, H. B., 2007: Advances in applications of the physics of fluids to severe weather systems. Rep. Prog. Phys., 70, 12591323, https://doi.org/10.1088/0034-4885/70/8/R01.

Boustead, J. M., and K. L. R. Gross, 2016: Environmental factors in the varying length of time between first echo and first tornado. 28th Conf. on Severe Local Storms, Portland, OR, Amer. Meteor. Soc., 49, https://ams.confex.com/ams/28SLS/ webprogram/Paper300651.html.

Church, C. R., J. T. Snow, G. L. Baker, and E. M. Agee, 1979: Characteristics of tornado-like vortices as a function of swirl ratio: A laboratory investigation. J. Atmos. Sci., 36, 1755-1776, https:// doi.org/10.1175/1520-0469(1979)036<1755:COTLVA > 2.0.CO;2.

Coffer, B. E., and M. D. Parker, 2016: Simulated supercells in nontornadic and tornadic VORTEX2 environments. Mon. Wea. Rev., 145, 149-180, https://doi.org/10.1175/MWR-D-160226.1 .

— and - 2018: Is there a "tipping point" between simulated nontornadic and tornadic supercells in VORTEX2 environments? Mon. Wea. Rev., 146, 2667-2693, https://doi.org/ 10.1175/MWR-D-18-0050.1. 
Dahl, J. M. L., M. D. Parker, and L. J. Wicker, 2012: Uncertainties in trajectory calculations within near-surface mesocyclones of simulated supercells. Mon. Wea. Rev., 140, 2959-2966, https:// doi.org/10.1175/MWR-D-12-00131.1.

Davies-Jones, R., 2015: A review of supercell and tornado dynamics. Atmos. Res., 158-159, 274-291, https://doi.org/ 10.1016/j.atmosres.2014.04.007.

, and H. Brooks, 1993: Mesocyclogenesis from a theoretical perspective. The Tornado: Its Structure, Dynamics, Prediction, and Hazards, Geophys. Monogr., Vol. 79, Amer. Geophys. Union, 105-114.

— currents in sheared environments. J. Atmos. Sci., 70, 12041215, https://doi.org/10.1175/JAS-D-12-0149.1.

Dawson, D. T., M. Xue, J. A. Milbrandt, and M. K. Yau, 2010: Comparison of evaporation and cold pool development between single-moment and multimoment bulk microphysics schemes in idealized simulations of tornadic thunderstorms. Mon. Wea. Rev., 138, 1152-1171, https://doi.org/10.1175/ 2009MWR2956.1.

_ B. Roberts, and M. Xue, 2019: A method to control the environmental wind profile in idealized simulations of deep convection with surface friction. Mon. Wea. Rev., 147, 39353954, https://doi.org/10.1175/MWR-D-18-0462.1.

Fiedler, B. H., 2017: Axisymmetric tornado simulations with a semi-slip boundary. Fluids, 2, 68, https://doi.org/10.3390/ fluids2040068.

Johnson, W. B., Jr., 1966: The "geotriptic" wind. Bull. Amer. Meteor. Soc., 47, 982, https://doi.org/10.1175/1520-0477-47.12.972.

Klemp, J. B., and R. Rotunno, 1983: A study of the tornadic region within a supercell thunderstorm. J. Atmos. Sci., 40, 359-377, https:// doi.org/10.1175/1520-0469(1983)040<0359:ASOTTR>2.0.CO;2.

Lemon, L. R., and C. A. Doswell, 1979: Severe thunderstorm evolution and mesocyclone structure as related to tornadogenesis. Mon. Wea. Rev., 107, 1184-1197, https://doi.org/ 10.1175/1520-0493(1979)107<1184:STEAMS>2.0.CO;2.

Leslie, F. W., 1977: Surface roughness effects on suction vortex formation: A laboratory simulation. J. Atmos. Sci., 34, 1022-1027, https://doi.org/10.1175/1520-0469(1977)034<1022: SREOSV $>2.0 . \mathrm{CO} ; 2$.

Lewellen, D. C., 2014: Local roughness effects on tornado dynamics. 27th Conf. on Severe Local Storms, Madison, WI, Amer. Meteor. Soc., 15A.1, https://ams.confex.com/ams/27SLS/ webprogram/Paper254357.html.

— W W. S. Lewellen, and J. Xia, 2000: The influence of a local swirl ratio on tornado intensification near the surface. J. Atmos. Sci., 57, 527-544, https://doi.org/10.1175/15200469(2000)057<0527:TIOALS >2.0.CO;2.

Lin, Y.-L., R. D. Farley, and H. D. Orville, 1983: Bulk parameterization of the snow field in a cloud model. J. Climate Appl. Meteor., 22, 1065-1092, https://doi.org/10.1175/1520-0450(1983) 022<1065:BPOTSF $>2.0$. CO;2.

Markowski, P. M., 2002: Hook echoes and rear-flank downdrafts: A review. Mon. Wea. Rev., 130, 852-876, https://doi.org/10.1175/ 1520-0493(2002)130<0852:HEARFD > 2.0.CO;2.

_ 2016: An idealized numerical simulation investigation of the effects of surface drag on the development of near-surface vertical vorticity in supercell thunderstorms. J. Atmos. Sci., 73, 4349-4385, https://doi.org/10.1175/JAS-D-16-0150.1.

, and G. H. Bryan, 2016: LES of laminar flow in the PBL: A potential problem for convective storm simulations. Mon. Wea. Rev., 144, 1841-1850, https://doi.org/10.1175/MWR-D15-0439.1.
_- Y. Richardson, E. Rasmussen, J. Straka, R. Davies-Jones, and R. J. Trapp, 2008: Vortex lines within low-level mesocyclones obtained from pseudo-dual-Doppler radar observations. Mon. Wea. Rev., 136, 3513-3535, https://doi.org/10.1175/ 2008MWR2315.1.

— N. T. Lis, D. D. Turner, T. R. Lee, and M. S. Buban, 2019: Observations of near-surface vertical wind profiles and vertical momentum fluxes from VORTEX-SE 2017: Comparisons to Monin-Obukhov similarity theory. Mon. Wea. Rev., 147, 3811-3824, https://doi.org/10.1175/MWR-D-19-0091.1.

Mashiko, W., 2016: A numerical study of the 6 May 2012 Tsukuba City supercell tornado. Part I: Vorticity sources of low-level and midlevel mesocyclones. Mon. Wea. Rev., 144, 1069-1092, https://doi.org/10.1175/MWR-D-15-0123.1.

Moeng, C.-H., and J. C. Wyngaard, 1988: Spectral analysis of largeeddy simulations of the convective boundary layer. J. Atmos. Sci., 45, 3573-3587, https://doi.org/10.1175/1520-0469(1988) 045<3573:SAOLES $>2.0 . \mathrm{CO} ; 2$.

Muñoz-Esparza, D., B. Kosović, J. Mirocha, and J. van Beeck, 2014: Bridging the transition from mesoscale to microscale turbulence in numerical weather prediction models. Bound.-Layer Meteor., 153, 409-440, https://doi.org/10.1007/ s10546-014-9956-9.

Noda, A. T., and H. Niino, 2010: A numerical investigation of a supercell tornado: Genesis and vorticity budget. J. Meteor. Soc. Japan, 88, 135-159, https://doi.org/10.2151/jmsj.2010-203.

Nolan, D. S., N. A. Dahl, G. H. Bryan, and R. Rotunno, 2017: Tornado vortex structure, intensity, and surface wind gusts in large-eddy simulations with fully developed turbulence. J. Atmos. Sci., 74, 1573-1597, https://doi.org/10.1175/JAS-D16-0258.1.

Palmer, R. D., and Coauthors, 2011: Observations of the 10 May 2010 tornado outbreak using OU-PRIME: Potential for new science with high-resolution polarimetric radar. Bull. Amer. Meteor. Soc., 92, 871-891, https://doi.org/10.1175/2011BAMS3125.1.

Reames, L. J., and D. J. Stensrud, 2018: Influence of a Great Plains urban environment on a simulated supercell. Mon. Wea. Rev., 146, 1437-1462, https://doi.org/10.1175/MWR-D-17-0284.1.

Roberts, B., 2017: The role of surface drag in supercell tornadogenesis and mesocyclogenesis: Studies based on idealized numerical simulations. Ph.D. dissertation, University of Oklahoma, $190 \mathrm{pp}$.

— intensification leading to tornadogenesis within an idealized supercell simulation. J. Atmos. Sci., 74, 3055-3077, https:// doi.org/10.1175/JAS-D-16-0364.1.

,-- A. D. Schenkman, and D. T. Dawson, 2016: The role of surface drag in tornadogenesis within an idealized supercell simulation. J. Atmos. Sci., 73, 3371-3395, https://doi.org/ 10.1175/JAS-D-15-0332.1.

Rotunno, R., 1977: Numerical simulation of a laboratory vortex. J. Atmos. Sci., 34, 1942-1956, https://doi.org/10.1175/15200469(1977)034<1942:NSOALV>2.0.CO;2.

_ , and J. Klemp, 1985: On the rotation and propagation of simulated supercell thunderstorms. J. Atmos. Sci., 42, 271-292, https:// doi.org/10.1175/1520-0469(1985)042<0271:OTRAPO>2.0.CO;2. , P. M. Markowski, and G. H. Bryan, 2017: "Near ground" vertical vorticity in supercell thunderstorm models. J. Atmos. Sci., 74, 1757-1766, https://doi.org/10.1175/JAS-D-16-0288.1.

Schenkman, A. D., M. Xue, and M. Hu, 2014: Tornadogenesis in a high-resolution simulation of the 8 May 2003 Oklahoma City supercell. J. Atmos. Sci., 71, 130-154, https://doi.org/10.1175/ JAS-D-13-073.1. 
Trapp, R. J., 2000: A clarification of vortex breakdown and tornadogenesis. Mon. Wea. Rev., 128, 888-895, https://doi.org/ 10.1175/1520-0493(2000)128<0888:ACOVBA > 2.0.CO;2.

— , G. R. Marion, and S. W. Nesbitt, 2017: The regulation of tornado intensity by updraft width. J. Atmos. Sci., 74, 41994211, https://doi.org/10.1175/JAS-D-16-0331.1.

Ward, N. B., 1972: The exploration of certain features of tornado dynamics using a laboratory model. J. Atmos. Sci., 29, 1194-1204, https://doi.org/10.1175/1520-0469(1972)029<1194: TEOCFO $>2.0 . \mathrm{CO} ; 2$.

Wieringa, J., 1993: Representative roughness parameters for homogeneous terrain. Bound.-Layer Meteor., 63, 323-363, https:// doi.org/10.1007/BF00705357.

Xue, M., K. K. Droegemeier, and V. Wong, 2000: The Advanced Regional Prediction System (ARPS) - A multi-scale nonhydrostatic atmospheric simulation and prediction model.
Part I: Model dynamics and verification. Meteor. Atmos. Phys., 75, 161-193, https://doi.org/10.1007/s007030070003.

, and Coauthors, 2001: The Advanced Regional Prediction System (ARPS) - A multi-scale nonhydrostatic atmospheric simulation and prediction tool. Part II: Model physics and applications. Meteor. Atmos. Phys., 76, 143-165, https:// doi.org/10.1007/s007030170027.

, D. Wang, J. Gao, K. Brewster, and K. K. Droegemeier, 2003: The Advanced Regional Prediction System (ARPS), storm-scale numerical weather prediction and data assimilation. Meteor. Atmos. Phys., 82, 139-170, https://doi.org/10.1007/s00703-001-0595-6.

Yokota, S., H. Niino, H. Seko, M. Kunii, and H. Yamauchi, 2018: Important factors for tornadogenesis as revealed by highresolution ensemble forecasts of the Tsukuba supercell tornado of 6 May 2012 in Japan. Mon. Wea. Rev., 146, 1109-1132, https://doi.org/10.1175/MWR-D-17-0254.1. 